\title{
Gromov-Witten invariants and integrable hierarchies of topological type
}

\author{
B.Dubrovin* \\ * SISSA, Trieste, and Steklov Mathematical Institute, Moscow \\ Dedicated to Sergei Petrovich Novikov \\ on the occasion of his birthday.
}

\begin{abstract}
We outline two approaches to the construction of integrable hierarchies associated with the theory of Gromov-Witten invariants of smooth projective varieties. We argue that a comparison of these two approaches yields nontrivial constraints on Chern numbers of varieties with semisimple quantum cohomology.
\end{abstract}

\section{Introduction}

The discovery of deep connections between topology of the Deligne-Mumford moduli spaces of stable algebraic curves and Korteweg-de Vries (KdV) hierarchy [48], [38, [45. [44], 37] revealed a new unexpected face of the theory of integrable systems. A program of describing the intersection theory on more general moduli spaces of stable maps of algebraic curves to smooth projective varieties in terms of suitable integrable hierarchies first outlined in [48] remains one of the main challenges on the way towards better understanding of relationships between the theory of integrable systems and topology. It was however soon realized that, except for few low-dimensional cases (see more details at the end of Section 3.3) the needed integrable hierarchies never appeared in the theory of integrable systems.

One arrives at the following question: given a smooth projective variety (or, more generally speaking, a compact symplectic manifold) $X$, can one construct a hierarchy of integrable partial differential equations (PDEs) such that the generating function of intersection numbers on the moduli stacks of stable maps of punctured algebraic curves to $X$ is given by (logarithm of) the tau-function of a particular solution to the hierarchy? An affirmative answer to this question was obtained in [22] under the assumption of semisimplicity of quantum cohomology of $X$. The basic idea of the construction that 
already appeared in [20] is very simple. Namely, we start with the genus zero hierarchy constructed in [11] (see also [49]) and, then, apply the so-called quasitriviality substitution changing the dependent variables of the hierarchy. The main question is how to find such a substitution. The original approach suggested in [20] uses the topological approach to the construction of the total descendent Gromov-Witten (GW) potential based on the study of identities [30] in the tautological ring of the Deligne-Mumford spaces (cf. [32]). An alternative approach of [22] uses expressions for the genus $g \geq 1$ total GW potential via the genus zero quantities derived from the Virasoro constraints, following the idea of [25]. In order to prove uniqueness of a solution to the system of Virasoro constraints the semisimplicity of quantum cohomology of $X$ was used in [22]

The main goal of this paper is to compare these two approaches. We show that the result of such a comparison are not completely trivial already in the study of the degree zero GW invariants, as it implies a constraint on the Chern numbers of a smooth projective variety with semisimple quantum cohomology (see Proposition 4.5 below and also [41, 25], 2]1).

The paper is organized as follows. In Section 2 we outline the basics of our construction of integrable hierarchies applying it to the well known example of the KdV hierarchy. Generalizing this construction we introduce in Section 3 an integrable hierarchy of topological type associated with an arbitrary smooth projective variety $X$ with $H^{\text {odd }}(X, \mathbb{C})=0$ having semisimple quantum cohomology, adapting to the topological setting the main ideas of [22]. The last Section 4 is dedicated to the comparison of results of the Section 3 with simple properties of moduli stacks of stable maps of degree zero.

\section{A construction of $\mathrm{KdV}$ hierarchy}

\subsection{KdV hierarchy from Lax representation}

It is well known that the Korteweg-de Vries $(\mathrm{KdV})$ equation for a function $u=$ $u(x, t)$

$$
u_{t}=u u_{x}+\frac{\epsilon^{2}}{12} u_{x x x}
$$

(the subscripts stand for partial derivatives) makes part of an infinite family of evolutionary partial differential equations (PDEs) for a function $u=u\left(x ; t_{0}, t_{1}, t_{2}, \ldots\right)$, the

\footnotetext{
${ }^{1}$ The author thanks Burt Totaro and Hsian-Hua Tseng for bringing his attention to the papers [4] and $[2]$.
} 
so-called $K d V$ hierarchy. The first few equations of the hierarchy read

$$
\begin{aligned}
& u_{t_{0}}=u_{x} \\
& u_{t_{1}}=u u_{x}+\frac{\epsilon^{2}}{12} u_{x x x} \\
& u_{t_{2}}=\frac{u^{2}}{2 !} u_{x}+\frac{\epsilon^{2}}{12}\left(2 u_{x} u_{x x}+u u_{x x x}\right)+\frac{\epsilon^{4}}{240} u^{V}
\end{aligned}
$$

etc. In a nutshell integrability of equations of the hierarchy means validity of the compatibility identities

$$
\left(u_{t_{i}}\right)_{t_{j}}=\left(u_{t_{j}}\right)_{t_{i}}
$$

for every pair of indices $i, j$ plus certain condition of completeness of the family of commuting flows (2.2) that we will not discuss here. The small parameter $\epsilon$ is responsible for dispersive effects of solutions to the $\mathrm{KdV}$ equation. Although it can be eliminated by rescaling $x \rightarrow \epsilon x, t_{k} \rightarrow \epsilon t_{k}$, we prefer to keep it as it will reappear below in the genus expansion of solutions to hierarchies of topological type.

There are various constructions of the KdV hierarchy. The most well known uses fractional powers of the Lax operator

$$
L=\frac{1}{2}\left(\epsilon \partial_{x}\right)^{2}+u(x)
$$

Define differential operators $A_{i}$ by the formula

$$
A_{i}=\frac{1}{(2 i+1) ! !}(2 L)_{+}^{\frac{2 i+1}{2}}=\frac{1}{(2 i+1) ! !}\left[\left(\epsilon \partial_{x}\right)^{2 i+1}+(2 i+1) u(x)\left(\epsilon \partial_{x}\right)^{2 i-1}+\ldots\right] .
$$

Here ()$_{+}$refers to the differential part of the pseudodifferential operator $(2 L)^{\frac{2 i+1}{2}}$. The $i$-th equation of the hierarchy admits the Lax representation

$$
u_{t_{i}} \equiv L_{t_{i}}=\left[A_{i}, L\right]
$$

\subsection{KdV hierarchy by a quasitriviality transformation}

Let us briefly outline one more construction of the KdV hierarchy, perhaps the one less known than others. Start with the so-called dispersionless limit of the equations (2.2)

$$
\begin{aligned}
& v_{t_{0}}=v_{x} \\
& v_{t_{1}}=v v_{x} \\
& v_{t_{2}}=\frac{v^{2}}{2 !} v_{x} \\
& \ldots \ldots \ldots \\
& v_{t_{i}}=\frac{v^{i}}{i !} v_{x}
\end{aligned}
$$


obtained by setting $\epsilon$ to zero (we have redenoted $u \rightarrow v$ the dependent function of the hierarchy for a later convenience). This is also an integrable hierarchy: validity of the compatibility identities

$$
\left(v_{t_{i}}\right)_{t_{j}}=\left(v_{t_{j}}\right)_{t_{i}}
$$

can be checked by a one line computation. We want to reconstruct the full hierarchy (2.2) from its dispersionless limit (2.7). To this end perform a substitution (the socalled quasitriviality transformation)

$$
v \mapsto u=v+\frac{\epsilon^{2}}{24}\left(\log v_{x}\right)_{x x}+\epsilon^{4}\left(\frac{v_{x x x x}}{1152 v_{x}^{2}}-\frac{7 v_{x x} v_{x x x}}{1920 v_{x}^{3}}+\frac{v_{x x}{ }^{3}}{360 v_{x}^{4}}\right)_{x x}+O\left(\epsilon^{6}\right)
$$

Let us explain the procedure at the first order in $\epsilon^{2}$. One has

$$
\begin{aligned}
& u_{t_{i}}=v_{t_{i}}+\frac{\epsilon^{2}}{24}\left(\frac{v_{x t_{i}}}{v_{x}}\right)_{x x}+\mathcal{O}\left(\epsilon^{4}\right)=\frac{\partial}{\partial x}\left[\frac{v^{i+1}}{(i+1) !}+\frac{\epsilon^{2}}{24}\left(\frac{v^{i}}{i !} \frac{v_{x x}}{v_{x}}+\frac{v^{i-1}}{(i-1) !} v_{x}\right)_{x}\right]+\ldots \\
& =\frac{\partial}{\partial x}\left[\frac{u^{i+1}}{(i+1) !}+\frac{\epsilon^{2}}{24}\left(2 \frac{u^{i-1}}{(i-1) !} u_{x x}+\frac{u^{i-2}}{(i-2) !} u_{x}^{2}\right)\right]+\mathcal{O}\left(\epsilon^{4}\right) .
\end{aligned}
$$

It can be easily checked that the resulting equation coincides with (2.6), within the $\epsilon^{2}$ approximation.

Integrability (2.3) of the full $\mathrm{KdV}$ hierarchy now readily follows from integrability (2.8) of the very simple hierarchy (2.7). Indeed, a change of variables preserves commutativity of the flows.

One can apply the same substitution to the bihamiltonian structure of the hierarchy (2.7). The latter is given by a compatible pair of Poisson brackets

$$
\begin{aligned}
& \{v(x), v(y)\}_{1}=\delta^{\prime}(x-y) \\
& \{v(x), v(y)\}_{2}=v(x) \delta^{\prime}(x-y)+\frac{1}{2} v_{x} \delta(x-y) .
\end{aligned}
$$

Recall that compatibility of the Poisson brackets means that the linear combination

$$
\{v(x), v(y)\}_{\lambda}:=\{v(x)-v(y)\}_{2}-\lambda\{v(x), v(y)\}_{1}
$$

is again a Poisson bracket for any $\lambda$. Equations of the hierarchy (2.7) can be represented in the Hamiltonian form in two different ways

$$
\begin{aligned}
v_{t_{i}} & =\left\{v(x), H_{i}\right\}_{1}=\partial_{x} \frac{\delta H_{i}}{\delta v(x)} \\
& =\left(i+\frac{1}{2}\right)^{-1}\left\{v(x), H_{i-1}\right\}_{2}=\left(v \partial_{x}+\frac{1}{2} v_{x}\right) \frac{\delta H_{i-1}}{\delta v(x)}
\end{aligned}
$$

where the Hamiltonian is defined by

$$
H_{i}=\int \frac{[v(x)]^{i+2}}{(i+2) !} d x .
$$


Applying the same substitution to the Poisson brackets (2.11), (2.12) and to the Hamiltonians (2.15) we arrive at a bihamiltonian structure of the full KdV hierarchy. Again, let us explain the idea of the calculation computing the first correction in $\epsilon^{2}$. Begin with the Hamiltonian. Within the $\mathcal{O}\left(\epsilon^{2}\right)$ order one has

$$
v=u-\frac{\epsilon^{2}}{24}\left(\log u_{x}\right)_{x x}+\mathcal{O}\left(\epsilon^{4}\right)
$$

So the Hamiltonian density $\frac{v^{i+2}}{(i+2) !}$ after the substitution becomes

$$
\begin{aligned}
& \frac{v^{i+2}}{(i+2) !}=\frac{u^{i+2}}{(i+2) !}-\frac{\epsilon^{2}}{24} \frac{u^{i+1}}{(i+1) !}\left(\log u_{x}\right)_{x x}+\mathcal{O}\left(\epsilon^{4}\right) \\
& =\frac{u^{i+2}}{(i+2) !}-\frac{\epsilon^{2}}{24}\left[\frac{u^{i-1}}{(i-1) !} u_{x}^{2}+\partial_{x}\left(\frac{u^{i+1}}{(i+1) !} \frac{u_{x x}}{u_{x}}-\frac{u^{i}}{i !} u_{x}\right)\right]+\mathcal{O}\left(\epsilon^{4}\right)
\end{aligned}
$$

The total $x$-derivative does not contribute to the Hamiltonian. Therefore

$$
H_{i}=\int\left[\frac{u^{i+2}}{(i+2) !}-\frac{\epsilon^{2}}{24} \frac{u^{i-1}}{(i-1) !} u_{x}^{2}\right] d x+\mathcal{O}\left(\epsilon^{4}\right) .
$$

Actually there is a more smart way for calculating the Hamiltonian densities that already eliminates all non-polynomial expressions. Besides the substitution (2.16) add a total derivative

$$
h_{i}:=\frac{v^{i+2}}{(i+2) !}+\epsilon^{2} \frac{\partial^{2} \Delta \mathcal{F}}{\partial x \partial t_{i+1}}=\frac{1}{(i+2) !}\left[u-\frac{\epsilon^{2}}{24}\left(\log u_{x}\right)_{x x}\right]^{i+2}+\frac{\epsilon^{2}}{24} \frac{\partial^{2} \log u_{x}}{\partial x \partial t_{i+1}}+\mathcal{O}\left(\epsilon^{4}\right) .
$$

Here

$$
\Delta \mathcal{F}=\frac{1}{24} \log v_{x}+\epsilon^{2}\left(\frac{v_{x x x x}}{1152 v_{x}^{2}}-\frac{7 v_{x x} v_{x x x}}{1920 v_{x}^{3}}+\frac{v_{x x}{ }^{3}}{360 v_{x}^{4}}\right)+O\left(\epsilon^{4}\right)
$$

(cf. (2.9) ). After simple calculation one obtains a polynomial, at this order, Hamiltonian density

$$
h_{i}=\frac{u^{i+2}}{(i+2) !}+\frac{\epsilon^{2}}{24}\left(2 \frac{u^{i} u_{x x}}{i !}+\frac{u^{i-1} u_{x}^{2}}{(i-1) !}\right)+\mathcal{O}\left(\epsilon^{4}\right) .
$$

The Hamiltonian $H_{i}=\int h_{i} d x$ coincides with the one obtained above but the densities differ by a total $x$-derivative. An advantage of the Hamiltonian densities $h_{i}=$ $h_{i}\left(u, u_{x}, \ldots ; \epsilon\right)$ defined by (2.17) $)^{2}$ is in validity of the following identities

$$
\frac{\partial h_{i-1}}{\partial t_{j}}=\frac{\partial h_{j-1}}{\partial t_{i}}
$$

\footnotetext{
${ }^{2} \mathrm{~A}$ construction of the Hamiltonian densities of the $\mathrm{KdV}$ hierarchy satisfying the tau-symmetry condition (2.18) in terms of the Hadamard-Seeley coefficients of the Lax operator (2.4) can be found in 22 .
} 
for arbitrary $i, j \geq 0$. Due to the tau-symmetry (2.18) one concludes that, for any common solution $u=u\left(x, t_{0}, t_{1}, \ldots\right)$ of the $\mathrm{KdV}$ hierarchy there exists a function $\tau=\tau\left(x, t_{0}, t_{1}, \ldots\right)$ such that

$$
h_{i}\left(u, u_{x}, \ldots\right)=\epsilon^{2} \frac{\partial^{2} \log \tau}{\partial x \partial t_{i+1}} .
$$

Such a representation makes sense also for $i=-1$ giving the trivial Hamiltonian

$$
h_{-1}=u=\epsilon^{2} \frac{\partial^{2} \log \tau}{\partial x^{2}}
$$

(the Casimir of the first Hamiltonian structure of the KdV hierarchy (see below)).

The transformation of the Poisson brackets can be calculated in a straightforward way. Let us apply the substitution (2.9) directly to the pencil (2.13) of Poisson brackets. One has

$$
\begin{aligned}
& \{u(x), u(y)\}_{\lambda}=\left\{v(x)+\frac{\epsilon^{2}}{24}\left(\log v_{x}\right)_{x x}, v(y)+\frac{\epsilon^{2}}{24}\left(\log v_{y}\right)_{y y}\right\}_{\lambda}+\ldots \\
& \{v(x), v(y)\}_{\lambda}+\frac{\epsilon^{2}}{24}\left[\left\{v(x),\left(\log v_{y}\right)_{y y}\right\}_{\lambda}+\left\{\left(\log v_{x}\right)_{x x}, v(y)\right\}_{\lambda}\right]+\ldots \\
& =\{v(x), v(y)\}_{\lambda}+\frac{\epsilon^{2}}{24}\left[\partial_{y}^{2} v_{y}^{-1} \partial_{y}\{v(x), v(y)\}_{\lambda}+\partial_{x}^{2} v_{x}^{-1} \partial_{x}\{v(x), v(y)\}_{\lambda}\right]+\ldots
\end{aligned}
$$

Using explicit expression for

$$
\{v(x), v(y)\}_{\lambda}=(v(x)-\lambda) \delta^{\prime}(x-y)+\frac{1}{2} v_{x} \delta(x-y)
$$

along with the general identity for derivatives of delta-function

$$
f(y) \delta^{(k)}(x-y)=f(x) \delta^{(k)}(x-y)+\sum_{j=1}^{k}\left(\begin{array}{c}
k \\
j
\end{array}\right) f^{(j)}(x) \delta^{(k-j)}(x-y)
$$

one rewrites the expression (2.20) in the form

$$
\begin{aligned}
& \{u(x), u(y)\}_{\lambda}=(v(x)-\lambda) \delta^{\prime}(x-y)+\frac{1}{2} v_{x} \delta(x-y)+\frac{\epsilon^{2}}{24}\left[\left(\log v_{x}\right)_{x x} \delta^{\prime}(x-y)\right. \\
& \left.+\frac{1}{2}\left(\log v_{x}\right)_{x x x} \delta(x-y)+3 \delta^{\prime \prime \prime}(x-y)\right]+\ldots \\
& =(u(x)-\lambda) \delta^{\prime}(x-y)+\frac{1}{2} u_{x} \delta(x-y)+\frac{\epsilon^{2}}{8} \delta^{\prime \prime \prime}(x-y)+\mathcal{O}\left(\epsilon^{4}\right) .
\end{aligned}
$$

It can be shown that terms of higher order in this expression all vanish.

Remark 2.1 The substitution (2.9) transforms solutions to equations of the dispersionless hierarchy (2.7) to those of the full KdV hierarchy (2.2). The resulting solution $u=u(x ; \mathbf{t} ; \epsilon)$ will be written as a series in $\epsilon^{2}$. However, it is applicable only to solutions of (2.7) monotone in $x$ due to presence of $v_{x}$ in the denominators.

\footnotetext{
${ }^{3}$ It turns out that higher jets $v^{(k)}$ for $k>1$ do not appear in the denominators.
} 


\subsection{Loop equation in the $\mathrm{KdV}$ theory}

Let us now outline the construction of the substitution (2.9). Define a sequence of second order linear differential operators $\mathcal{L}_{m}, m \geq-1$ with coefficients depending on a parameter $\epsilon$ acting on functions of jet variables $v \equiv v^{(0)}, v_{x} \equiv v^{(1)}, v_{x x} \equiv v^{(2)}, \ldots$, $v^{(k)}, \ldots$. They are conveniently defined by the following generating function

$$
\sum_{m=-1}^{\infty} \frac{\mathcal{L}_{m}}{\lambda^{m+2}}=\sum_{k \geq 0}\left(A_{k}(\lambda)-\epsilon^{2} B_{k}(\lambda)\right) \frac{\partial}{\partial v^{(k)}}-\frac{\epsilon^{2}}{2} \sum_{k, l \geq 0} C_{k l}(\lambda) \frac{\partial^{2}}{\partial v^{(k)} \partial v^{(l)}}+\frac{1}{16(v-\lambda)^{2}}
$$

where

$$
\begin{aligned}
& A_{k}(\lambda)=A_{k}\left(\lambda ; v, v_{x}, \ldots, v^{(k)}\right)=\partial_{x}^{k}\left(\frac{1}{v-\lambda}\right)+\sum_{j=1}^{k}\left(\begin{array}{c}
k \\
j
\end{array}\right) \partial_{x}^{j-1}\left(\frac{1}{\sqrt{v-\lambda}}\right) \partial_{x}^{k-j+1}\left(\frac{1}{\sqrt{v-\lambda}}\right) \\
& B_{k}(\lambda)=B_{k}\left(\lambda ; v, v_{x}, \ldots, v^{(k+2)}\right)=-\frac{1}{16} \partial_{x}^{k+2}\left(\frac{1}{(v-\lambda)^{2}}\right) \\
& C_{k l}(\lambda)=C_{k l}\left(\lambda ; v, v_{x}, \ldots, v^{(\max (k, l)+1)}\right)=\partial_{x}^{k+1}\left(\frac{1}{\sqrt{v-\lambda}}\right) \partial_{x}^{l+1}\left(\frac{1}{\sqrt{v-\lambda}}\right)
\end{aligned}
$$

and the operator $\partial_{x}$ is defined by

$$
\partial_{x}=\sum_{k \geq 0} v^{(k+1)} \frac{\partial}{\partial v^{(k)}} .
$$

Clearly the coefficients $A_{k}(\lambda), B_{k}(\lambda), C_{k l}(\lambda)$ are rational functions of $\lambda$ with poles only at $\lambda=v$. They depend polynomially on the jets $v_{x}, v_{x x}$ etc. Explicitly,

$$
\begin{aligned}
& \mathcal{L}_{-1}=-\frac{\partial}{\partial v} \\
& \mathcal{L}_{0}=-\sum_{k \geq 0} \frac{2 k+1}{2} v^{(k)} \frac{\partial}{\partial v^{(k)}}+\frac{1}{16}
\end{aligned}
$$

etc.

Lemma 2.2 The operators (2.22) satisfy commutation relations

$$
\left[\mathcal{L}_{i}, \mathcal{L}_{j}\right]=(i-j) \mathcal{L}_{i+j}, \quad i, j \geq-1 .
$$

We are now ready to write a system of equations defining the substitution (2.9).

Theorem 2.3 There exists a unique, up to additive constants, sequence of functions $\mathcal{F}_{g}\left(v, v_{x}, \ldots, v^{(3 g-2)}\right)$ satisfying the following system of equations

$$
\mathcal{L}_{m} e^{\Delta \mathcal{F}}=0, \quad m \geq-1
$$

where

$$
\Delta \mathcal{F}:=\sum_{g \geq-1} \epsilon^{2 g-2} \mathcal{F}_{g}\left(v, v_{x}, \ldots, v^{(3 g-2)}\right)
$$


The equations (2.26) yield

$$
\mathcal{F}_{1}=\frac{1}{24} \log v_{x}
$$

and then an overdetermined system of recursion relations

$$
\sum_{k=0}^{3 g-2} A_{k}(\lambda) \frac{\partial \mathcal{F}_{g}}{\partial v^{(k)}}=\sum_{k=0}^{3 g-5} B_{k}(\lambda) \frac{\partial \mathcal{F}_{g-1}}{\partial v^{(k)}}+\frac{1}{2} \sum_{k, l=0}^{3 g-5} C_{k l}(\lambda)\left(\frac{\partial^{2} \mathcal{F}_{g-1}}{\partial v^{(k)} \partial v^{(l)}}+\sum_{h=1}^{g-1} \frac{\partial \mathcal{F}_{h}}{\partial v^{(k)}} \frac{\partial \mathcal{F}_{g-h}}{\partial v^{(l)}}\right)
$$

depending on the parameter $\lambda$. Compatibility of the resulting overdetermined linear system essentially follows from commutation relations (2.25). So one obtains

$$
\mathcal{F}_{2}=\frac{v_{x x x x}}{1152 v_{x}{ }^{2}}-\frac{7 v_{x x} v_{x x x}}{1920 v_{x}{ }^{3}}+\frac{v_{x x}{ }^{3}}{360 v_{x}{ }^{4}}
$$

etc.

The last step is the following

Theorem 2.4 After the substitution

$$
v \mapsto u=v+\epsilon^{2} \partial_{x}^{2} \Delta \mathcal{F}
$$

the dispersionless hierarchy (2.7) transforms to the KdV hierarchy (2.2), (2.6). Here $\Delta \mathcal{F}$ is the solution to the system (2.26).

\section{Integrable hierarchies of topological type}

In this section the construction of integrable hierarchies of topological type will be adapted to the environment of Gromov-Witten (GW) invariants we will begin with.

\subsection{Gromov-Witten invariants of a smooth projective vari- eties, their descendents and total Gromov-Witten poten- tial}

Let $X$ be a smooth projective variety of complex dimension $d$. For simplicity it will be assumed that

$$
H^{\text {odd }}(X, \mathbb{C})=0 .
$$

Denote $n=\operatorname{dim} H^{*}(X, \mathbb{C})$. Choose a basis $\gamma_{1}=1, \gamma_{2}, \ldots, \gamma_{n}$ of cohomologies. Assume

$$
\gamma_{\alpha} \in H^{2 q_{\alpha}}(X), \quad q_{1}=0, \quad q_{n}=d .
$$

The class $\gamma_{n} \in H^{2 d}(X)$ coincides with the volume element normalized by

$$
\int_{X} \gamma_{n}=1
$$


The Poincaré pairing on the cohomology will be denoted by

$$
\langle a, b\rangle=\int_{X} a \wedge b
$$

Denote

$$
\eta_{\alpha \beta}=\int_{X} \gamma_{\alpha} \wedge \gamma_{\beta}
$$

the Gram matrix of the pairing with respect to the basis $\gamma_{1}, \ldots, \gamma_{n}$. We will use this symmetric matrix and its inverse $\eta^{\alpha \beta}$ for lowering and raising indices in the subsequent formulae.

GW invariants and their descendents can be defined as intersection numbers of certain cycles on the moduli stack $\bar{M}_{g, m}(X, \beta)$ of stable maps

$$
\bar{M}_{g, m}(X, \beta)=\left\{f:\left(C_{g} ; x_{1}, \ldots, x_{m}\right) \rightarrow X, \quad f_{*}\left[C_{g}\right]=\beta\right\} / \text { equivalencies }
$$

of a given degree $\beta \in H_{2}(X ; \mathbb{Z}) /$ torsion. Here $C_{g}$ is an algebraic curve of genus $g$ with at most double points singularities, $x_{1}, \ldots, x_{m}$ are pairwise distinct marked points on $C_{g}$. Equivalencies are isomorphisms of $C_{g} \rightarrow X$ identical on $X$ and on the markings. Put

$$
\left\langle\tau_{p_{1}}\left(\gamma_{\alpha_{1}}\right) \ldots \tau_{p_{m}}\left(\gamma_{\alpha_{m}}\right)\right\rangle_{g, m, \beta}=\int_{\left[\bar{M}_{g, m}(X, \beta)\right]^{\mathrm{virt}}} \mathrm{ev}_{1}^{*}\left(\gamma_{\alpha_{1}}\right) \psi_{1}^{p_{1}} \ldots \mathrm{ev}_{m}^{*}\left(\gamma_{\alpha_{m}}\right) \psi_{m}^{p_{m}}
$$

Here

$$
\mathrm{ev}_{i}: \bar{M}_{g, m}(X, \beta) \rightarrow X, \quad f \mapsto f\left(x_{i}\right)
$$

are evaluation maps,

$$
\psi_{i}=c_{1}\left(\mathcal{L}_{i}\right), \quad \text { Chern class of tautological line bundle } \underset{M_{g, m}(X, \beta)}{\mid \mathcal{L}_{i}}
$$

Finally, the virtual fundamental class $\left[\bar{M}_{g, m}(X, \beta)\right]^{\text {virt }}$ is an element of the Chow ring $A_{*}\left(\bar{M}_{g, m}(X, \beta)\right)$

$$
\left[\bar{M}_{g, m}(X, \beta)\right]^{\mathrm{virt}} \in A_{D}\left(\bar{M}_{g, m}(X, \beta)\right), \quad D=(1-g)(d-3)+m+\left\langle\beta, c_{1}(X)\right\rangle .
$$

Note that, due to effectivity (see [42]) it suffices to consider the invariants (3.7) only for cycles in the semigroup

$$
\beta \in B(X) \subset H_{2}(X ; \mathbb{Z}) / \text { torsion, } \quad \int_{\beta} \omega \geq 0 \quad \forall \omega \in \text { Kähler cone } \subset H^{2}(X) .
$$

Generating function of rational numbers (3.7) (also called total descendent potential of genus $g$ ) for a given genus $g$ is defined by the series

$$
\mathcal{F}_{g}(\mathbf{t}, q)=\sum_{m} \sum_{\left(\alpha_{1}, p_{1}\right), \ldots,\left(\alpha_{m}, p_{m}\right)} \frac{t_{p_{1}}^{\alpha_{1}} \ldots t_{p_{m}}^{\alpha_{m}}}{m !} \sum_{\beta \in H_{2}(X ; \mathbb{Z})}\left\langle\tau_{p_{1}}\left(\gamma_{\alpha_{1}}\right) \ldots \tau_{p_{m}}\left(\gamma_{\alpha_{m}}\right)\right\rangle_{g, m, \beta} q^{\beta}
$$


Here $t_{p}^{\alpha}$ are indeterminates, $\alpha=1, \ldots, n, p=0,1, \ldots$;

$$
q^{\beta}=q_{1}^{m_{1}} \ldots q_{l}^{m_{l}} \quad \text { for } \quad \beta=m_{1} \beta_{1}+\cdots+m_{l} \beta_{l}
$$

where $\beta_{1}, \ldots \beta_{l}$ is a basis in $H_{2}(X ; \mathbb{Z}) /$ torsion

is an element of the Novikov ring. The total GW potential is defined by summation over genera,

$$
\mathcal{F}(\mathbf{t}, q, \epsilon)=\sum_{g=0}^{\infty} \epsilon^{2 g-2} \mathcal{F}_{g} .
$$

The additional parameter $\epsilon$ of the genus expansion is called string coupling constant in physics literature. It will play the role of small dispersion expansion parameter in the integrable hierarchy we are going to construct under certain additional assumptions.

More specifically, our goal is to construct a hierarchy of integrable PDEs with $n$ dependent functions and time variables $\mathbf{t}=\left(t_{p}^{\alpha}\right), t_{0}^{1}=x$, such that

$$
\tau(\mathbf{t}, q, \epsilon)=e^{\mathcal{F}}
$$

is tau-function of a particular solution to the hierarchy.

In general it is hard to think about reconstruction of differential equations starting from just one solution. However, the design of construction explained in the previous section for the particular case of $\mathrm{KdV}$ hierarchy suggests a very natural recipe we are going to outline in this section.

The clue is in using the Frobenius structure on the manifold $M=H^{*}(X, \mathbb{C})$ defined by the genus zero $G W$ potential with no $\psi$-classes 4 . Introduce affine coordinates $v^{1}$, $\ldots, v^{n}$ on this space associated with the chosen basis $\gamma_{1}, \ldots, \gamma_{n}$. Denote

$$
\mathbf{v}=v^{\alpha} \gamma_{\alpha} \in H^{*}(X, \mathbb{C})
$$

(summation over repeated indices here and below will be assumed). Put

$$
F(\mathbf{v}, q)=\sum_{m} \sum_{\alpha_{1}, \ldots, \alpha_{m}} \frac{v^{\alpha_{1}} \ldots v^{\alpha_{m}}}{m !} \sum_{\beta \in H_{2}(X ; \mathbb{Z})} \int_{\bar{M}_{0, m}(X, \beta)} \operatorname{ev}_{1}^{*}\left(\gamma_{\alpha_{1}}\right) \ldots \mathrm{ev}_{m}^{*}\left(\gamma_{\alpha_{m}}\right) q^{\beta}
$$

Due to divisor axiom [42] the dependence on $q=\left(q_{1}, \ldots, q_{l}\right)$ can be essentially eliminated by a shift

$$
\begin{aligned}
& v^{\alpha_{i}} \mapsto v^{\alpha_{i}}-\sum_{s=1}^{l} M_{i s} \log q_{s}, \quad i=1, \ldots, l=\operatorname{dim} H^{2}(X), \quad M_{i s}=\int_{\beta_{s}} \gamma_{\alpha_{i}} \\
& \gamma_{\alpha_{1}}, \ldots, \gamma_{\alpha_{l}} \quad \text { a basis in } H^{2}(X), \quad \beta_{1}, \ldots, \beta_{l} \quad \text { a basis of the lattice } H_{2}(X ; \mathbb{Z}) / \text { torsion }
\end{aligned}
$$

\footnotetext{
${ }^{4}$ No descendents, in physics terminology.
} 
under assumption of convergence. Such convergence will be assumed in sequel. So the dependence on $q=\left(q_{1}, \ldots, q_{l}\right)$ is often omitted from the formulae of the theory of Frobenius manifolds. Triple derivatives of this function

$$
c_{\alpha \beta}^{\gamma}(\mathbf{v})=\eta^{\gamma \delta} \frac{\partial^{3} F(\mathbf{v})}{\partial v^{\delta} \partial v^{\alpha} \partial v^{\beta}}
$$

where

$$
\left(\eta^{\alpha \beta}\right)=\left(\eta_{\alpha \beta}\right)^{-1}
$$

define on $M$ a structure of Frobenius manifold, i.e., a structure of commutative associative algebra on the tangent space $T_{\mathbf{v}} M$ at every point $\mathbf{v} \in M$,

$$
\frac{\partial}{\partial v^{\alpha}} \cdot \frac{\partial}{\partial v^{\beta}}=c_{\alpha \beta}^{\gamma}(\mathbf{v}) \frac{\partial}{\partial v^{\gamma}}
$$

a flat invariant metric

$$
\left\langle\frac{\partial}{\partial v^{\alpha}}, \frac{\partial}{\partial v^{\beta}}\right\rangle=\eta_{\alpha \beta}
$$

a unit

$$
e=\frac{\partial}{\partial v^{1}}
$$

and an Euler vector field

$$
E=\sum_{\alpha=1}^{n}\left[\left(1-q_{\alpha}\right) v^{\alpha}+\left\langle c_{1}(X), \gamma^{\alpha}\right\rangle\right] \frac{\partial}{\partial v^{\alpha}}
$$

entering into the quasihomogeneity property of the potential $F$

$$
E F=(3-d) F+\text { quadratic polynomial. }
$$

\subsection{Genus zero invariants and Principal Hierarchy}

For any function $f(\mathbf{t})$ of infinite number of variables $t_{p}^{\alpha}$ define an operation of restriction on the small phase space by

$$
\left.f(\mathbf{t})\right|_{\text {small phase space }}:=\left.f(\mathbf{t})\right|_{t_{0}^{\alpha}=v^{\alpha}, t_{p}^{\alpha}=0 \text { for } p>0, \alpha=1, \ldots, n} .
$$

Thus, for example

$$
F(\mathbf{v}, q)=\left.\mathcal{F}_{0}(\mathbf{t}, q)\right|_{\text {small phase space }}
$$

Define functions $\theta_{\alpha, p}(\mathbf{v}, q)$ by

$$
\theta_{\alpha, p}(\mathbf{v}, q):=\left.\frac{\partial^{2} \mathcal{F}_{0}(\mathbf{t}, q)}{\partial t_{0}^{1} \partial t_{0}^{\alpha}}\right|_{\text {small phase space. }}
$$

The generating functions

$$
\theta_{\alpha}(\mathbf{v}, q ; z)=\sum_{p=0}^{\infty} \theta_{\alpha, p}(\mathbf{v}, q) z^{p}, \quad \alpha=1, \ldots, n
$$


form a basis of horizontal sections

$$
\tilde{\nabla}(z) d \theta_{\alpha}(\mathbf{v}, q ; z)=0
$$

of the canonical deformed flat connection

$$
\tilde{\nabla}_{a}(z) b=\nabla_{a} b+z a \cdot b, \quad a, b \text { are vector fields on } \quad M .
$$

A choice of such a basis is called calibration of the Frobenius manifold $M$. In the GW setting we will always choose the calibration in the form (3.23), (3.24).

We are ready to define Principal Hierarchy associated with a calibrated Frobenius manifold as a system of PDEs for vector-valued functions $\mathbf{v}=\mathbf{v}(x, \mathbf{t})$ taking values in $M=H^{*}(X)$. Equations of the hierarchy read

$$
\frac{\partial \mathbf{v}}{\partial t_{p}^{\alpha}}=\frac{\partial}{\partial x} \nabla \theta_{\alpha, p+1}(\mathbf{v}, q), \quad \alpha=1, \ldots, n, \quad p \geq 0 .
$$

In particular

$$
\frac{\partial}{\partial t_{0}^{1}}=\frac{\partial}{\partial x}
$$

The equation (3.27) is a system of $n$ Hamiltonian PDEs with the Hamiltonian

$$
H_{\alpha, p}^{0}=\int \theta_{\alpha, p+1}(\mathbf{v}(x)) d x
$$

and Poisson bracket

$$
\left\{v^{\alpha}(x), v^{\beta}(y)\right\}_{1}=\eta^{\alpha \beta} \delta^{\prime}(x-y) .
$$

Moreover, the equations of the principal hierarchy commute pairwise,

$$
\left\{H_{\alpha, p}^{0}, H_{\beta, q}^{0}\right\}_{1}=0 \Rightarrow \frac{\partial}{\partial t_{p}^{\alpha}} \frac{\partial \mathbf{v}}{\partial t_{q}^{\beta}}=\frac{\partial}{\partial t_{q}^{\beta}} \frac{\partial \mathbf{v}}{\partial t_{p}^{\alpha}}=0 .
$$

They also admit a second Hamiltonian structure defined by the Poisson bracket

$$
\begin{aligned}
& \left\{v^{\alpha}(x), v^{\beta}(y)\right\}_{2}=g^{\alpha \beta}(\mathbf{v}(x)) \delta^{\prime}(x-y)+\Gamma_{\gamma}^{\alpha \beta}(\mathbf{v}(x)) \delta(x-y) \\
& g^{\alpha \beta}(\mathbf{v})=E^{\gamma}(\mathbf{v}) c_{\gamma}^{\alpha \beta}(\mathbf{v}), \quad \Gamma_{\gamma}^{\alpha \beta}(\mathbf{v})=\left(\frac{1}{2}-\mu_{\beta}\right) c_{\gamma}^{\alpha \beta}(\mathbf{v}) .
\end{aligned}
$$

Here

$$
\mu_{\beta}=q_{\beta}-\frac{d}{2}
$$

The symmetric matrix $g^{\alpha \beta}(\mathbf{v})$ is called intersection form of the Frobenius manifold. It does not degenerate on an open dense subset in $M$. So, according to [18] its inverse defines another flat metric on this subset. Moreover, the pair of symmetric matrices $g^{\alpha \beta}(\mathbf{v})$ and $\eta^{\alpha \beta}$ form a so-called flat pencil (see details in [13]). Existence of such a flat pencil is essentially equivalent to the axioms of Frobenius manifold [ibid]. Flat coordinates of the linear combination

$$
g^{\alpha \beta}(\mathbf{v})-\lambda \eta^{\alpha \beta}
$$

will play an important role in the constructions of the next section. 
Example 3.1 For $X=$ pt one obtains one-dimensional Frobenius manifold; all structure constants (3.15) are constants. The function $\theta(v, z)$ can be found from (3.25)

$$
\theta(v, z)=\frac{e^{z v}-1}{z}=\sum_{p=1}^{\infty} \frac{v^{p+1}}{(p+1) !} z^{p} .
$$

So, in this case the principal hierarchy coincides with (2.7).

Any solution $\mathbf{v}=\mathbf{v}(\mathbf{t})$ to the principal hierarchy can be represented in implicit form as follows. Consider a function $\Phi_{\mathbf{t}}(\mathbf{v})$ on the Frobenius manifold $M$ depending on the infinite vector of time variables $\mathbf{t}=\left(t_{p}^{\alpha}\right)$ defined as follows

$$
\Phi_{\mathbf{t}}(\mathbf{v})=\sum_{p \geq 0} t_{p}^{\alpha} \theta_{\alpha, p}(\mathbf{v})
$$

Choose a constant vector $\mathbf{t}_{0}=\left(t_{p_{0}}^{\alpha}\right)$ and consider a system of $n$ equations written in the vector form

$$
\nabla \Phi_{\mathbf{t}}(\mathbf{v})=\nabla \Phi_{\mathbf{t}_{0}}(\mathbf{v})
$$

Suppose that the system (3.36) satisfies conditions of the implicit function theorem near the point $\mathbf{t}=0$. Then the solution to (3.36) can be expanded in a formal power series in $\left(t_{p}^{\alpha}\right)_{p>0}$. The resulting series satisfy equations (3.27). All solutions to the hierarchy (3.27) satisfying certain genericity conditions can be obtained in such a way. Such an important statement is usually referred to as completeness of the family of commuting Hamiltonian flows (3.27).

Choose a solution $\mathbf{v}(\mathbf{t})$ in the form of $(3.36)$ and define a function $\mathcal{F}_{0}(\mathbf{t})$ by integrating the following closed 1 -form on $M$

$$
\mathcal{F}_{0}(\mathbf{t})=\left.\frac{1}{2} \int d \Phi_{\mathbf{t}-\mathbf{t}_{0}}(\mathbf{v}) \cdot d \Phi_{\mathbf{t}-\mathbf{t}_{0}}(\mathbf{v})\right|_{\mathbf{v}=\mathbf{v}(\mathbf{v}(\mathbf{t})} .
$$

In this formula the product of 1-forms is understood in the sense of the dual Frobenius algebra structure defined on the cotangent space $T^{*} M$ (we refer to [12] for details about choice of integration constants in (3.37) $)$. The function

$$
\tau_{0}(\mathbf{t})=\exp \frac{1}{\epsilon^{2}} \mathcal{F}_{0}(\mathbf{t})
$$

is called the tau-function of the solution (3.36) to the principal hierarchy. The solution itself can be expressed via the second logarithmic derivatives of the tau-function

$$
v_{\alpha}(\mathbf{t})=\epsilon^{2} \frac{\partial^{2}}{\partial x \partial t_{0}^{\alpha}} \log \tau_{0}(\mathbf{t}), \quad \alpha=1, \ldots, n .
$$

Moreover, the density of the Hamiltonian $H_{\alpha, p-1}^{0}$ evaluated on the solution $\mathbf{v}(\mathbf{t})$ can also represented as certain second logarithmic derivatives of the tau-function

$$
\theta_{\alpha, p}(\mathbf{v}(\mathbf{t}))=\epsilon^{2} \frac{\partial^{2}}{\partial x \partial t_{p}^{\alpha}} \log \tau_{0}(\mathbf{t})
$$


For the particular choice of the shifts $\mathbf{t}_{0}$ given by

$$
t_{1_{0}}^{1}=1, \quad t_{p_{0}}^{\alpha}=0 \quad \text { otherwise }
$$

the function $\mathcal{F}_{0}(\mathbf{t})$ coincides with the genus zero $\mathrm{GW}$ total descendent potential function (3.12). For this reasons the solution $\mathbf{v}(\mathbf{t})$ specified by the shifts (3.41) will be called topological solution to the principal hierarchy. It will be denoted by $\mathbf{v}_{\text {top }}(\mathbf{t})$

Example 3.2 For $X=$ pt the topological solution $v=v_{\text {top }}(\mathbf{t})$ is determined by the equation

$$
x+\sum_{p=0}^{\infty} t_{p} \frac{v^{p}}{p !}=v
$$

The series expansion of this solution (3.42) reads

$$
v_{\text {top }}(\mathbf{t})=\sum_{n=1}^{\infty} \frac{1}{n} \sum_{p_{1}+\cdots+p_{n}=n-1} \frac{t_{p_{1}}}{p_{1} !} \ldots \frac{t_{p_{n}}}{p_{n} !} .
$$

The (logarithm of) the tau-function (3.37), (3.38) of this solution is given by the formula

$$
\mathcal{F}_{0}(\mathbf{t})=\sum_{n=3}^{\infty} \frac{1}{n(n-1)(n-2)} \sum_{p_{1}+\cdots+p_{n}=n-3} \frac{t_{p_{1}}}{p_{1} !} \ldots \frac{t_{p_{n}}}{p_{n} !} .
$$

It coincides with the generating function of intersection numbers of $\psi$-classes on the moduli spaces $\overline{\mathcal{M}}_{0, n}$ of Riemann spheres with $n$ punctures (see more details in the next section below)

$$
\mathcal{F}_{0}(\mathbf{t})=\sum_{n=3}^{\infty} \frac{1}{n !} \sum_{p_{1}, \ldots, p_{n}} t_{p_{1}} \ldots t_{p_{n}} \int_{\overline{\mathcal{M}}_{0, n}} \psi_{1}^{p_{1}} \ldots \psi_{n}^{p_{n}}
$$

\subsection{Loop equations and construction of integrable hierarchy of topological type associated with a smooth projective variety with semisimple quantum cohomology}

Let us now explain the procedure of reconstructing of the full hierarchy written in the form of an infinite $\epsilon$-expansion starting from the Principal Hierarchy associated with a calibrated semisimple Frobenius manifold of dimension $n$. As above we will assume that the Frobenius manifold is realized as the quantum cohomology of a smooth projective variety $X$. Assumption of semisimplicity is a highly nontrivial restriction onto the class of varieties (see more on the problem of semisimplicity in [14], [1], [35], [36], [47], [26], [46]).

Lemma 3.3 Under assumption of semisimplicity the deformed flat coordinates (3.23), (3.24) are entire functions of the variable $z \in \mathbb{C}$. 
We will consider $\left(\theta_{1}(\mathbf{v} ; z), \ldots, \theta_{n}(\mathbf{v} ; z)\right)$ as a $H_{*}(X)$-valued function on $M \times \mathbb{C}$. Define twisted periods $p_{\alpha}^{(\nu)}(\mathbf{v} ; \lambda)$ by a Laplace-type integral

$$
\left(p_{1}^{(\nu)}(\mathbf{v} ; \lambda), \ldots, p_{n}^{(\nu)}(\mathbf{v} ; \lambda)\right):=\int_{0}^{\infty} \frac{d z}{z^{\frac{1}{2}+\nu}} e^{-\lambda z}\left(\theta_{1}(\mathbf{v} ; z), \ldots, \theta_{n}(\mathbf{v} ; z)\right) z^{\mu} z^{c_{1}(X)} .
$$

Here

$$
z^{c_{1}(X)}=1+\frac{c_{1}(X)}{1 !} \log z+\frac{c_{1}^{2}(X)}{2 !} \log ^{2} z+\cdots+\frac{c_{1}^{d}(X)}{d !} \log ^{d} z
$$

is a polynomial in $\log z$. The obvious formula

$$
\int_{0}^{\infty} e^{-s} s^{x-1} \log ^{k} s d s=\Gamma^{(k)}(x), \quad-x \notin \mathbb{Z} .
$$

can be used in order to give sense to the integral (3.46) for $\operatorname{Re} \nu<<0$. The definition can be extended to all complex values of $\nu$ satisfying

$$
\nu \notin \operatorname{Spec} \mu+\mathbb{Z}_{\geq 0}-\frac{1}{2}
$$

The resulting twisted periods are analytic functions on the universal covering of

$$
M \times \mathbb{C} \backslash\{\operatorname{det}(\mathcal{U}(\mathbf{v})-\lambda)=0\}
$$

where $\mathcal{U}(\mathbf{v})$ is the operator of quantum multiplication by the Euler vector field $E(\mathbf{v})$, see details in [15]. If the complex dimension $d=\operatorname{dim}_{\mathbb{C}} X$ is even then the twisted periods are well defined for $\nu=0$. In this case $p_{1}^{(0)}(\mathbf{v} ; \lambda), \ldots, p_{n}^{(0)}(\mathbf{v} ; \lambda)$ are flat coordinates for the $\lambda$-dependent metric (3.33).

Introduce matrix $G(\nu)=\left(G^{\alpha \beta}(\nu)\right)$ by

$$
G(\nu)=-\frac{1}{2 \pi}\left(e^{\pi i c_{1}(X)} e^{\pi i(\mu-\nu)}+e^{-\pi i c_{1}(X)} e^{-\pi i(\mu-\nu)}\right) \eta^{-1}
$$

and define coefficients $A_{k}^{\gamma}=A_{k}^{\gamma}\left(\mathbf{v} ; \mathbf{v}_{x}, \ldots, \mathbf{v}^{(k)} ; \lambda\right), B_{k}^{\gamma}=B_{k}^{\gamma}\left(\mathbf{v} ; \mathbf{v}_{x}, \ldots, \mathbf{v}^{(k+2)} ; \lambda\right)$, $C_{k l}^{\gamma \rho}=C_{k l}^{\gamma \rho}\left(\mathbf{v} ; \mathbf{v}_{x}, \ldots, \mathbf{v}^{(\max (k, l)+1)} ; \lambda\right), k, l=0,1,2, \ldots$, by the following formulae

$$
\begin{aligned}
& A_{k}^{\gamma}=\partial_{x}^{k}\left((E(\mathbf{v})-\lambda e)^{-1}\right)^{\gamma}+\lim _{\nu \rightarrow 0} \sum_{j=1}^{k}\left(\begin{array}{c}
k \\
j
\end{array}\right) \partial_{x}^{j-1} \nabla_{1} p_{\alpha}^{(-\nu)}(\mathbf{v} ; \lambda) G^{\alpha \beta}(\nu) \partial_{x}^{k-j+1} \nabla^{\gamma} p_{\beta}^{(\nu)}(\mathbf{v} ; \lambda) \\
& B_{k}^{\gamma}=\frac{1}{2} \partial_{x}^{k+1}\left[\left(\lim _{\nu \rightarrow 0} G^{\alpha \beta}(\nu) \partial_{\lambda} \nabla p_{\alpha}^{(-\nu)}(\mathbf{v} ; \lambda) \cdot \partial_{\lambda} \nabla p_{\beta}^{(\nu)}(\mathbf{v} ; \lambda)\right) \cdot \mathbf{v}_{x}\right]^{\gamma} \\
& C_{k l}^{\gamma \rho}=\lim _{\nu \rightarrow 0} \partial_{x}^{k+1} \nabla^{\gamma} p_{\alpha}^{(-\nu)}(\mathbf{v} ; \lambda) G^{\alpha \beta}(\nu) \partial_{x}^{l+1} \nabla^{\rho} p_{\beta}^{(\nu)}(\mathbf{v} ; \lambda) .
\end{aligned}
$$

In these formulae all Greek indices take their values from 1 to $n$; summation over repeated Greek indices is always assumed. The product and inversion of vector fields on $M$ in the expressions for $B_{k}^{\gamma}$ and $A_{k}^{\gamma}$ are understood in the sense of the quantum multiplication on $T M$. 
Theorem 3.4 1. The coefficients (3.50) are well defined.

2. They are rational functions in $\lambda$ vanishing at $\lambda=\infty$ having poles at $\lambda \in \operatorname{Spec} \mathcal{U}(\mathbf{v})$.

3. Introduce $\epsilon$-dependent linear differential operators $\mathcal{L}_{m}, m \geq-1$ acting on functions of jet variables

$$
v^{\alpha, k}:=\partial_{x}^{k} v^{\alpha}, \quad \alpha=1, \ldots, n, \quad k=0,1,2, \ldots
$$

by the generating series

$$
\begin{aligned}
& \sum_{m \geq-1} \frac{\mathcal{L}_{m}}{\lambda^{m+2}}= \\
& =\sum_{k \geq 0}\left(A_{k}^{\gamma}-\epsilon^{2} B_{k}^{\gamma}\right) \frac{\partial}{\partial v^{\gamma, k}}-\frac{\epsilon^{2}}{2} \sum_{k, l} C_{k l}^{\gamma \rho} \frac{\partial^{2}}{\partial v^{\gamma, k} \partial v^{\rho, l}}+\frac{1}{16} \operatorname{tr}(\mathcal{U}(\mathbf{v})-\lambda)^{-2}-\frac{1}{4} \operatorname{tr}\left[(\mathcal{U}(\mathbf{v})-\lambda)^{-1} \mu\right]^{2} .
\end{aligned}
$$

In this formula the operator $\mu: H^{*}(X) \rightarrow H^{*}(X)$ is defined by

$$
\mu=\frac{\operatorname{deg}-d}{2}
$$

These operators satisfy commutation relations

$$
\left[\mathcal{L}_{i}, \mathcal{L}_{j}\right]=(i-j) \mathcal{L}_{i+j}, \quad i, j \geq-1
$$

4. Consider a system of the so-called loop equations

$$
\mathcal{L}_{k} e^{\sum_{g \geq 1} \epsilon^{2 g-2} \mathcal{F}_{g}\left(\mathbf{v} ; \mathbf{v}_{x}, \mathbf{v}_{x x}, \ldots, \mathbf{v}^{(3 g-2)}\right)}=0, \quad k \geq-1
$$

for functions $\mathcal{F}_{1}\left(\mathbf{v} ; \mathbf{v}_{x}\right), \mathcal{F}_{2}\left(\mathbf{v} ; \mathbf{v}_{x}, \mathbf{v}_{x x}, \mathbf{v}_{x x x}, \mathbf{v}_{x x x x}\right)$ etc. For any smooth projective variety $X$ with semisimple quantum cohomology the system of equations (3.54) has a unique, up to additive constants $\mathcal{F}_{g} \mapsto \mathcal{F}_{g}+c_{g}$, solution.

The proof can be derived from results of [22].

Equations (3.54) yield a linear inhomogeneous system

$$
A_{0}^{\gamma} \frac{\partial \mathcal{F}_{1}}{\partial v^{\gamma}}+A_{1}^{\gamma} \frac{\partial \mathcal{F}_{1}}{\partial v_{x}^{\gamma}}=-\frac{1}{16} \operatorname{tr}(\mathcal{U}(\mathbf{v})-\lambda)^{-2}+\frac{1}{4} \operatorname{tr}\left[(\mathcal{U}(\mathbf{v})-\lambda)^{-1} \mu\right]^{2}
$$

for the first derivatives of the function $\mathcal{F}_{1}=\mathcal{F}_{1}\left(\mathbf{v} ; \mathbf{v}_{x}\right)$ along with a recursion relation

$$
\sum_{k=0}^{3 g-2} A_{k}^{\gamma} \frac{\partial \mathcal{F}_{g}}{\partial v^{\gamma, k}}=\sum_{k=0}^{3 g-5} B_{k}^{\gamma} \frac{\partial \mathcal{F}_{g-1}}{\partial v^{\gamma, k}}+\frac{1}{2} \sum_{k, l=0}^{3 g-5} C_{k l}^{\gamma \rho}\left(\frac{\partial^{2} \mathcal{F}_{g-1}}{\partial v^{\gamma, k} \partial v^{\rho, l}}+\sum_{h=1}^{g-1} \frac{\partial \mathcal{F}_{h}}{\partial v^{\gamma, k}} \frac{\partial \mathcal{F}_{g-h}}{\partial v^{\rho, l}}\right)
$$

for $g \geq 2$.

The above construction of the Virasoro operators $\mathcal{L}_{i}$ is closely related to a Sugawaratype construction of [22]. Let us briefly summarize the latter. 
Introduce the Heisenberg algebra with the generators

$$
a_{\alpha, p}, \quad \alpha=1, \ldots, n, \quad p \in \mathbb{Z}+\frac{1}{2}
$$

and the commutation relations

$$
\left[a_{\alpha, p}, a_{\beta, q}\right]=(-1)^{p-\frac{1}{2}} \eta_{\alpha \beta} \delta_{p+q, 0} .
$$

Introduce the row vectors

$$
\mathbf{a}_{p}=\left(a_{1, p}, \ldots, a_{n, p}\right)
$$

and their generating function

$$
\mathbf{a}(z)=\sum \mathbf{a}_{p} z^{p}
$$

Define

$$
\phi_{\alpha}^{(\nu)}(\lambda)=\left(\int_{0}^{\infty} \frac{d z}{z^{1-\nu}} e^{-\lambda z} \mathbf{a}(z) z^{\mu} z^{c_{1}(X)}\right)_{\alpha}, \quad \alpha=1, \ldots, n .
$$

Put

$$
T^{(\nu)}(\lambda)=\sum_{m \in \mathbf{Z}} \frac{L_{m}^{(\nu)}}{\lambda^{m+2}}=-\frac{1}{2}: \partial_{\lambda} \phi_{\alpha}^{(-\nu)} G^{\alpha \beta}(\nu) \partial_{\lambda} \phi_{\beta}^{(\nu)}:+\frac{1}{4 \lambda^{2}} \operatorname{tr}\left(\frac{1}{4}-\mu^{2}\right)
$$

where the normal ordering is defined by

$$
\begin{array}{ll}
: a_{\alpha, p} a_{\beta, q}:=a_{\beta, q} a_{\alpha, p} & \text { if } q<0, p>0, \\
: a_{\alpha, p} a_{\beta, q}:=a_{\alpha, p} a_{\beta, q} & \text { otherwise. }
\end{array}
$$

Here $\nu$ is a complex parameter.

Lemma 3.5 [22] Let $k$ be the minimal positive integer such that $c_{1}(X)^{k}=0$. Then there exist the limits

$$
\begin{aligned}
L_{m} & :=\lim _{\nu \rightarrow 0} L_{m}^{(\nu)}, \quad m \geq-1 \\
L_{m} & :=\lim _{\nu \rightarrow 0} \nu^{k} L_{m}^{(\nu)}, \quad m<-1 .
\end{aligned}
$$

These operators satisfy the following commutation relations

$$
\begin{aligned}
& {\left[L_{i}, L_{j}\right]=0, \quad i, j<-1, \text { or } i+j \geq-1, \text { but }(i+1)(j+1)<0} \\
& {\left[L_{i}, L_{j}\right]=(i-j) L_{i+j}, \quad i+j<-1, \quad(i+1)(j+1)<0, \quad \text { or } i, j \geq-1 .}
\end{aligned}
$$

A natural representation of the Heisenberg algebra (3.57) is obtained as follows

$$
\begin{aligned}
& a_{\alpha, p}=\epsilon \frac{\partial}{\partial t_{p-\frac{1}{2}}^{\alpha}}, \quad p>0, \\
& a_{\alpha, p}=\epsilon^{-1}(-1)^{p+\frac{1}{2}} \eta_{\alpha \beta} t_{-p-\frac{1}{2}}^{\beta}, \quad p<0 .
\end{aligned}
$$


In this representation the operators $L_{m}$ for $m \geq-1$ become linear second order differential operators

$$
\begin{aligned}
& L_{m}=L_{m}\left(\epsilon^{-1} \mathbf{t}, \epsilon \partial / \partial \mathbf{t}\right) \\
& =\epsilon^{2} \sum a_{m}^{\alpha, p ; \beta, q} \frac{\partial^{2}}{\partial t^{\alpha, p} \partial t^{\beta, q}}+\sum b_{m \beta, q}^{\alpha, p} t^{\beta, q} \frac{\partial}{\partial t^{\alpha, p}}+\epsilon^{-2} c_{\alpha, p ; \beta, q}^{m} t^{\alpha, p} t^{\beta, q}+\frac{1}{4} \delta_{m, 0} \operatorname{tr}\left(\frac{1}{4}-\mu^{2}\right)
\end{aligned}
$$

for some constant coefficients $a_{m}^{\alpha, p ; \beta, q}, b_{m, \beta}^{\alpha, p}, c_{\alpha, p ; \beta, q}^{m}$ depending on $m \geq-1$ and on the spectrum of $\mu$ and on the first Chern class $c_{1}(X)$.

We are now ready to complete the construction of integrable hierarchy of topological type associated with a smooth projective variety with semisimple quantum cohomology. Solve the system of Virasoro constraints and then apply the substitution

$$
v_{\alpha} \mapsto u_{\alpha}=v_{\alpha}+\sum_{g \geq 1} \epsilon^{2 g} \frac{\partial^{2} \mathcal{F}_{g}\left(\mathbf{v} ; \mathbf{v}_{x}, \mathbf{v}_{x x}, \ldots, \mathbf{v}^{(3 g-2)}\right)}{\partial x \partial t_{0}^{\alpha}}, \quad \alpha=1, \ldots, n
$$

to the principal hierarchy (3.27). We arrive at the following main construction [22].

Theorem 3.6 1) The resulting hierarchy

$$
\frac{\partial u_{\alpha}}{\partial t_{p}^{\beta}}=\frac{\partial v_{\alpha}}{\partial t_{p}^{\beta}}+\sum_{g \geq 1} \epsilon^{2 g} \frac{\partial^{3} \mathcal{F}_{g}\left(\mathbf{v} ; \mathbf{v}_{x}, \mathbf{v}_{x x}, \ldots, \mathbf{v}^{(3 g-2)}\right)}{\partial x \partial t_{0}^{\alpha} \partial t_{p}^{\beta}}, \quad \alpha, \beta=1, \ldots, n, \quad p \geq 0
$$

admits a hamiltonian description

$$
\frac{\partial u_{\alpha}}{\partial t_{p}^{\beta}}=P_{\alpha \gamma} \frac{\delta H_{\beta, p}}{\delta u_{\gamma}(x)}
$$

with the first Poisson bracket given by the operator

$$
P_{\alpha \beta}=\eta_{\lambda \mu} \mathrm{L}_{\alpha}^{\lambda} \circ \partial_{x} \circ \mathrm{L}_{\beta}^{* \mu}
$$

where the matrix-valued operator $\mathrm{L}_{\alpha}^{\beta}$ is the linearization of the substitution (3.64),

$$
\mathrm{L}_{\alpha}^{\beta}=\delta_{\alpha}^{\beta}+\sum_{k \geq 0} \frac{\partial}{\partial u_{\beta}^{(k)}}\left(\sum_{g \geq 1} \epsilon^{2 g} \frac{\partial^{2} \mathcal{F}_{g}\left(\mathbf{v} ; \mathbf{v}_{x}, \mathbf{v}_{x x}, \ldots, \mathbf{v}^{(3 g-2)}\right)}{\partial x \partial t_{0}^{\alpha}}\right) \frac{\partial^{k}}{\partial x^{k}},
$$

$\mathrm{L}_{\alpha}^{* \beta}$ is the formal adjoint operator to $\mathrm{L}_{\alpha}^{\beta}$. The Hamiltonians of the hierarchy with respect to the first Poisson structure have the form

$$
\begin{aligned}
& H_{\beta, p}=\int h_{\beta, p}\left(\mathbf{u} ; \mathbf{u}_{x}, \mathbf{u}_{x x}, \ldots ; \epsilon\right) d x \\
& h_{\beta, p}\left(\mathbf{u} ; \mathbf{u}_{x}, \mathbf{u}_{x x}, \ldots ; \epsilon\right)=\theta_{\beta, p+1}(\mathbf{v})+\sum_{g \geq 1} \epsilon^{2 g} \frac{\partial^{2} \mathcal{F}_{g}\left(\mathbf{v} ; \mathbf{v}_{x}, \mathbf{v}_{x x}, \ldots, \mathbf{v}^{(3 g-2)}\right)}{\partial x \partial t_{p+1}^{\beta}} .
\end{aligned}
$$


The functions $h_{\beta,-1}=u_{\beta}$ are densities of Casimirs of the first Poisson bracket. The Hamiltonian densities (3.69) satisfy the tau-symmetry equations

$$
\frac{\partial h_{\alpha, p-1}}{\partial t_{q}^{\beta}}=\frac{\partial h_{\beta, q-1}}{\partial t_{p}^{\alpha}}, \quad \alpha, \beta=1, \ldots, n, \quad p, q=0,1,2, \ldots
$$

2) The flows (3.66) are also bihamiltonian as they preserve another Poisson bracket defined by the operator $\tilde{P}_{\alpha \beta}$ obtained by a similar transformation applied to (3.32). The brackets $P_{\alpha \beta}$ and $\tilde{P}_{\alpha \beta}$ are compatible.

3) Given a solution $\mathbf{v}(\mathbf{t})$ of the Principal hierarchy, the function $\mathbf{u}(\mathbf{t} ; \epsilon)$ defined by (3.64) satisfies the hierarchy (3.66). The tau-function of this solution reads

$$
\begin{aligned}
& \tau(\mathbf{t} ; \epsilon)=\exp \left[\frac{1}{\epsilon^{2}} \mathcal{F}_{0}(\mathbf{t})+\sum_{g \geq 1} \epsilon^{2 g-2} \mathcal{F}_{g}\left(\mathbf{v}(\mathbf{t}) ; \mathbf{v}_{x}(\mathbf{t}), \mathbf{v}_{x x}(\mathbf{t}), \ldots, \mathbf{v}^{(3 g-2)}(\mathbf{t})\right)\right] \\
& u_{\alpha}(\mathbf{t} ; \epsilon)=\epsilon^{2} \frac{\partial^{2} \log \tau(\mathbf{t} ; \epsilon)}{\partial x \partial t_{0}^{\alpha}}, \quad \alpha=1, \ldots, n .
\end{aligned}
$$

Here the genus zero tau-function is defined by (3.37), (3.38).

4) For $m \geq-1$ linear action of Virasoro operators $L_{m}$ defined in Lemma 3.5 on tau-functions generates infinitesimal symmetries of the hierarchy (3.66), i.e., given a tau-function $\tau=\tau(\mathbf{t} ; \epsilon)$ of a solution to the hierarchy (3.66), then for an arbitrary small parameter $\delta$ the functions

$$
u_{\alpha}(\mathbf{t} ; \epsilon ; \delta)=\epsilon^{2} \frac{\partial^{2} \log \left(\tau+\delta \cdot L_{m} \tau\right)}{\partial x \partial t_{0}^{\alpha}}
$$

for any $m \geq-1$ satisfy, modulo corrections of order $\mathcal{O}\left(\delta^{2}\right)$, the same equations of the hierarchy (3.66) .

5) Denote $\mathbf{u}_{\mathrm{top}}(\mathbf{t} ; \epsilon)$ the solution to the hierarchy (3.66) obtained from the topological solution $\mathbf{v}_{\text {top }}(\mathbf{t})$ to the principal hierarchy (see above). Let $\tau_{\text {top }}(\mathbf{t} ; \epsilon)$ be the tau-function (3.71) of $\mathbf{u}_{\mathrm{top}}(\mathbf{t} ; \epsilon)$. It satisfies the Virasoro constraints

$$
L_{m}\left(\epsilon^{-1}\left(\mathbf{t}-\mathbf{t}_{0}\right), \epsilon \partial / \partial \mathbf{t}\right) \tau_{\text {top }}(\mathbf{t} ; \epsilon)=0, \quad m \geq-1 .
$$

Corollary 3.7 Assuming validity of the Virasoro conjecture [25] for the variety $X$, the logarithm of the topological tau-function $\tau_{\text {top }}(\mathbf{t} ; \epsilon)$ coincides with the total $G W$ potential (3.13).

Example 3.8 For $X=$ pt the twisted periods are

$$
p^{(\nu)}(v ; \lambda)=\Gamma\left(-\frac{1}{2}-\nu\right)(\lambda-v)^{\nu+\frac{1}{2}}
$$


and the $1 \times 1$ matrix (3.49) reads

$$
G(\nu)=-\frac{1}{\pi} \cos \pi \nu
$$

One can easily see that the general formulae (3.50)-(3.56) reduce to (2.22)-(2.29). Actually in this case one can replace in (3.50) from the very beginning $p^{( \pm \nu)}$ by $p^{(0)}=$ $\sqrt{v-\lambda}$ (up to a constant factor). Observe that $\sqrt{v-\lambda}$ coincides with the flat coordinate of the flat pencil of metrics (3.33), i.e., of the $\lambda$-dependent metric

$$
\frac{d v^{2}}{v-\lambda}
$$

obtained by inversion of (3.33). The topological tau-function $\tau_{\text {top }}(\mathbf{t} ; \epsilon)$ coincides with the Witten-Kontsevich tau-function of the KdV hierarchy. It gives the generating function of intersection numbers of $\psi$-classes on the Deligne-Mumford moduli spaces $\overline{\mathcal{M}}_{g, n}$.

The Virasoro symmetries of the KdV hierarchy are generated by the following linear operators

$$
\begin{aligned}
L_{m} & =\frac{\epsilon^{2}}{2} \sum_{k+l=m-1} \frac{(2 k+1) ! !(2 l+1) ! !}{2^{m+1}} \frac{\partial^{2}}{\partial t_{k} \partial t_{l}} \\
& +\sum_{k \geq 0} \frac{(2 k+2 m+1) ! !}{2^{m+1}(2 k-1) ! !} t_{k} \frac{\partial}{\partial t_{k+m}}+\frac{1}{16} \delta_{m, 0}, \quad m \geq 0 \\
L_{-1} & =\sum_{k \geq 1} t_{k} \frac{\partial}{\partial t_{k-1}}+\frac{1}{2 \epsilon^{2}} t_{0}^{2}
\end{aligned}
$$

The Witten-Kontsevich tau-function $\tau=\tau_{\text {top }}(\mathbf{t})$ is uniquely specified by the system of linear equations

$$
\begin{aligned}
& L_{m} \tau=\frac{(2 m+3) ! !}{2^{m+1}} \partial_{m+1} \tau, \quad m \geq 0 \\
& L_{-1} \tau=\partial_{0} \tau
\end{aligned}
$$

Example 3.9 For $X=\mathbf{P}^{1}$ the basis is $\gamma_{1}=1, \gamma_{2}=\omega \in H^{2}\left(\mathbf{P}^{1}\right)$,

$$
\int_{\mathbf{P}^{1}} \omega=1 .
$$

In order to simplify notations we will redenote

$$
v^{1} \rightarrow v, \quad v^{2} \rightarrow u
$$

the corresponding flat coordinates on the two-dimensional Frobenius manifold $M=$ $H^{*}\left(\mathbf{P}^{1}\right)$. So the notation

$$
\mathbf{v}=v+u \omega \in M
$$


will still be used for a generic point in the Frobenius manifold. The potential of the Frobenius structure, the Gram matrix of the flat metric, the unity, and the Euler vector field read

$$
F=\frac{1}{2} v^{2} u+e^{u}, \quad \eta=\left(\begin{array}{cc}
0 & 1 \\
1 & 0
\end{array}\right), \quad e=\frac{\partial}{\partial v}, \quad E=v \frac{\partial}{\partial v}+2 \frac{\partial}{\partial u} .
$$

Thus the operator of multiplication by E has the following matrix

$$
\mathcal{U}(\mathbf{v})=\left(\begin{array}{cc}
v & 2 e^{u} \\
2 & v
\end{array}\right)
$$

The deformed flat coordinates can be expressed via modified Bessel functions

$$
\begin{aligned}
& \theta_{1}(\mathbf{v} ; z)=\sum_{p \geq 0} \theta_{1, p}(v, u) z^{p}=-2 e^{z v}\left(K_{0}\left(2 z e^{\frac{1}{2} u}\right)+(\log z+\gamma) I_{0}\left(2 z e^{\frac{1}{2} u}\right)\right) \\
& \theta_{2}(\mathbf{v} ; z)=\sum_{p \geq 0} \theta_{2, p}(v, u) z^{p}=z^{-1}\left[e^{z v} I_{0}\left(2 z e^{\frac{1}{2} u}\right)-1\right]
\end{aligned}
$$

Here $\gamma$ is the Euler-Mascheroni constant. The time variables of the Principal Hierarchy will be redenoted

$$
t_{k}^{1} \rightarrow t_{k}, \quad t_{k}^{2} \rightarrow s_{k}
$$

So

$$
\begin{array}{ll}
\frac{\partial v}{\partial t_{k}}=\frac{\partial}{\partial x} \frac{\partial \theta_{1, k+1}(v, u)}{\partial u}, & \frac{\partial u}{\partial t_{k}}=\frac{\partial}{\partial x} \frac{\partial \theta_{1, k+1}(v, u)}{\partial v} \\
\frac{\partial v}{\partial s_{k}}=\frac{\partial}{\partial x} \frac{\partial \theta_{2, k+1}(v, u)}{\partial u}, & \frac{\partial u}{\partial s_{k}}=\frac{\partial}{\partial x} \frac{\partial \theta_{2, k+1}(v, u)}{\partial v}
\end{array}
$$

In particular,

$$
\frac{\partial v}{\partial s_{0}}=e^{u} u_{x}, \quad \frac{\partial u}{\partial s_{0}}=v_{x}
$$

A basis of twisted periods, after a suitable linear combination, can be expressed via Legendre function 5 of the 1 st and 2 nd kind

$$
\begin{aligned}
& p_{1}^{(\nu)}(\mathbf{v} ; \lambda)=\frac{1}{\nu} e^{-\frac{u}{4}} \Delta^{\frac{1}{4}+\frac{\nu}{2}} P_{-\frac{1}{2}}^{\frac{1}{2}+\nu}(w) \\
& p_{2}^{(\nu)}(\mathbf{v} ; \lambda)=\frac{1}{\nu} e^{-\frac{u}{4}} \Delta^{\frac{1}{4}+\frac{\nu}{2}} Q_{-\frac{1}{2}}^{\frac{1}{2}+\nu}(w)
\end{aligned}
$$

where

$$
\Delta=-\operatorname{det}(\mathcal{U}(\mathbf{v})-\lambda)=4 e^{u}-(v-\lambda)^{2}, \quad w=\frac{1}{2} e^{-\frac{u}{2}}(\lambda-v) .
$$

\footnotetext{
${ }^{5}$ Also called Ferrers functions.
} 
The matrix (3.49) in this basis becomes

$$
G(\nu)=\left(\begin{array}{cc}
\frac{\pi}{2} \cos \pi \nu & -\sin \pi \nu \\
\sin \pi \nu & \frac{2}{\pi} \cos \pi \nu
\end{array}\right)
$$

For the coefficients (3.50) one easily obtains

$$
\begin{aligned}
& A_{0}^{1}=\frac{\lambda-v}{\Delta}, \quad A_{0}^{2}=\frac{2}{\Delta} \\
& A_{1}^{1}=-\frac{8 e^{u}+(v-\lambda)^{2}}{\Delta^{2}} v_{x}+\frac{6 e^{u}(v-\lambda)}{\Delta^{2}} u_{x}, \quad A_{1}^{2}=\frac{6(v-\lambda)}{\Delta^{2}} v_{x}-\frac{12 e^{u}}{\Delta^{2}} u_{x} .
\end{aligned}
$$

Furthermore, since $\mu=\operatorname{diag}\left(-\frac{1}{2}, \frac{1}{2}\right)$ we have

$$
\operatorname{tr}(\mathcal{U}(\mathbf{v})-\lambda)^{-2}=2 \frac{4 e^{u}+(v-\lambda)^{2}}{\Delta^{2}}, \quad \operatorname{tr}\left[(\mathcal{U}(\mathbf{v})-\lambda)^{-1} \mu\right]^{2}=-\frac{1}{2 \Delta}
$$

So the equation (3.55) yields

$$
\mathcal{F}_{1}=\frac{1}{24}\left[\log \left(v_{x}^{2}-e^{u} u_{x}^{2}\right)-u\right] .
$$

This formula was derived in [20] from topological considerations.

A somewhat more lengthy computation allows one to also derive higher genus terms.

For example (cf. 24]),

$$
\begin{aligned}
& 5760 \mathcal{F}_{2}= \\
& =-\frac{q^{2}}{D^{4}}\left[512 u_{x}^{3} v_{x} v_{x x}^{3}+384 q u_{x}^{3} v_{x x}\left(u_{x}^{2}+2 u_{x x}\right)\left(u_{x}^{2} v_{x}+2 u_{x x} v_{x}-2 u_{x} v_{x x}\right)-64 q^{2} u_{x}^{4}\left(u_{x}^{2}+2 u_{x x}\right)^{3}\right] \\
& -\frac{q}{D^{3}}\left[256 u_{x} v_{x} v_{x x}^{3}+12 q u_{x}\left(28 u_{x}^{4} v_{x} v_{x x}+116 u_{x}^{2} u_{x x} v_{x} v_{x x}+64 u_{x x}^{2} v_{x} v_{x x}+28 u_{x} v_{x} u_{x x x} v_{x x}-69 u_{x}^{3} v_{x x}^{2}\right.\right. \\
& \left.-128 u_{x} u_{x x} v_{x x}^{2}+14 u_{x}^{3} v_{x} v_{x x x}+28 u_{x} v_{x} u_{x x} v_{x x x}-28 u_{x}^{2} v_{x x} v_{x x x}\right) \\
& \left.-q^{2} u_{x}^{2}\left(u_{x}^{2}+2 u_{x x}\right)\left(121 u_{x}^{4}+538 u_{x}^{2} u_{x x}+256 u_{x x}^{2}+168 u_{x} u_{x x x}\right)\right] \\
& +\frac{q}{D^{2}}\left[-2\left(42 u_{x}^{3} v_{x} v_{x x}+126 u_{x} u_{x x} v_{x} v_{x x}+42 u_{x x x} v_{x} v_{x x}-95 u_{x}^{2} v_{x x}^{2}-96 u_{x x} v_{x x}^{2}+30 u_{x}^{2} v_{x} v_{x x x}\right.\right. \\
& \left.+42 u_{x x} v_{x} v_{x x x}-126 u_{x} v_{x x} v_{x x x}+20 u_{x} v_{x} v_{x x x x}\right)+q\left(72 u_{x}^{6}+479 u_{x}^{4} u_{x x}+626 u_{x}^{2} u_{x x}^{2}+64 u_{x x}^{3}\right. \\
& \left.\left.+224 u_{x}^{3} u_{x x x}+252 u_{x} u_{x x} u_{x x x}+40 u_{x}^{2} u_{x x x x}\right)\right]-\frac{1}{D}\left[22 v_{x x}^{2}-24 v_{x} v_{x x x}+q\left(17 u_{x}^{4}+102 u_{x}^{2} u_{x x}\right.\right. \\
& \left.\left.+56 u_{x x}^{2}+68 u_{x} u_{x x x}+20 u_{x x x x}\right)\right]+7 u_{x x}
\end{aligned}
$$

where we denote

$$
q=e^{u}, \quad D=v_{x}^{2}-e^{u} u_{x}^{2} .
$$

Applying the quasitriviality substitution

$$
\begin{aligned}
& u \mapsto u+\epsilon^{2} \partial_{x}^{2} \Delta \mathcal{F} \\
& v \mapsto v+\epsilon^{2} \partial_{x} \partial_{s_{0}} \Delta \mathcal{F}
\end{aligned}
$$


where the $s_{0}$-derivatives are defined by (3.74) and

$$
\Delta \mathcal{F}=\mathcal{F}_{1}+\epsilon^{2} \mathcal{F}_{2}+\ldots
$$

one obtains the extended Toda hierarchy [6], [23]. The latter can be represented in the Lax form

$$
\begin{aligned}
& \epsilon \frac{\partial L}{\partial s_{k}}=\frac{1}{(k+1) !}\left[\left(L^{k+1}\right)_{+}, L\right] \\
& \epsilon \frac{\partial L}{\partial t_{k}}=\frac{2}{k !}\left[L^{k}\left(\log L-c_{k}\right)_{+}, L\right], \quad c_{k}=1+\frac{1}{2}+\cdots+\frac{1}{k} .
\end{aligned}
$$

Here $L$ is a degree two difference operator acting on functions on the $x$-axis by

$$
L=\Lambda+v(x)+e^{u(x)} \Lambda^{-1}
$$

where $\Lambda$ stands for the shift operator

$$
\Lambda f(x)=f(x+\epsilon)
$$

the symbol ( ) + refers to the part of a difference operator containing only nonnegative degrees $\Lambda^{k}, k \geq 0$. We refer the reader to [6] for the definition of logarithm of a difference operator.

The Virasoro symmetries of the extended Toda hierarchy are generated by the following linear operators

$$
\begin{aligned}
& L_{m}=\epsilon^{2} \sum_{k=1}^{m-1} k !(m-k) ! \frac{\partial^{2}}{\partial s_{k-1} \partial s_{m-k-1}} \\
& +\sum_{k \geq 1} \frac{(m+k) !}{(k-1) !}\left(t_{k} \frac{\partial}{\partial t_{m+k}}+s_{k-1} \frac{\partial}{\partial s_{m+k-1}}\right)+2 \sum_{k \geq 0} \alpha_{m}(k) t_{k} \frac{\partial}{\partial s_{m+k-1}}, \quad m>0 \\
& L_{0}=\sum_{k \geq 1} k\left(t_{k} \frac{\partial}{\partial t_{k}}+s_{k-1} \frac{\partial}{\partial s_{k-1}}\right)+\sum_{k \geq 1} 2 t_{k} \frac{\partial}{\partial s_{k-1}}+\frac{1}{\epsilon^{2}} t_{0}^{2}, \\
& L_{-1}=\sum_{k \geq 1}\left(t_{k} \frac{\partial}{\partial t_{k-1}}+s_{k} \frac{\partial}{\partial s_{k-1}}\right)+\frac{1}{\epsilon^{2}} t_{0} s_{0} .
\end{aligned}
$$

Here the integer coefficients $\alpha_{m}(k)$ are defined by

$$
\alpha_{m}(0)=m !, \quad \alpha_{m}(k)=\frac{(m+k) !}{(k-1) !}[\psi(k+m+1)-\psi(k)], \quad k>0
$$

where $\psi(x)$ is the digamma function. 
For other smooth projective varieties $X$ with semisimple quantum cohomology very little is known about the associated integrable hierarchies of topological type. The conjectural description of the integrable hierarchy of topological type associated with the orbifold quantum cohomology of $\mathbf{P}^{1}$ with two orbifold points is given by the socalled bigraded Toda hierarchy [43], [7]. A somewhat more general setting of GW theory of the resolved conifold $\mathcal{O}_{\mathbf{P}^{1}}(-1) \oplus \mathcal{O}_{\mathbf{P}^{1}}(-1)$ with respect to the antidiagonal torus action has been recently studied in [3] in connection with the Ablowitz-Ladik integrable hierarchy. A one-parameter deformation of the KdV hierarchy closely connected with the intermediate long wave equation was recently obtained in [4] by inserting certain combinations of Hodge $\lambda$-classes into the Witten-Kontsevich generating series. Except for these examples and also Drinfeld-Sokolov hierarchies of $A D E$ type [10], [17], [28], [50] related to computation of intersection numbers on the moduli spaces of algebraic curves with higher spin structures [49] and their generalizations [29] other integrable hierarchies of topological type arising in the quantum cohomology setting seem to be unknown in the theory of integrable systems.

Remark 3.10 In [33] a so-called total descendent potential was associated with an arbitrary calibrated semisimple Frobenius manifold. Identifying Virasoro constraints of [33] with those constructed in [22] (see above the explicit formulae) one can prove that the Givental's total descendent potential is equal to the logarithm of the tau-function of the topological solution to the integrable hierarchy of topological type associated with the Frobenius manifold.

\subsection{On axiomatic approach to integrable hierarchies of topo- logical type}

What can be said about properties of the integrable hierarchies of topological type constructed by Theorem 3.6 above? Clearly, they remain integrable since they are obtained by change of dependent variables from the integrable principle hierarchy. Moreover, applying the same quasitriviality substitution to the bihamiltonian structure of the principal hierarchy one obtains the bihamiltonian structure for the resulting hierarchy of topological type. For example, we will write here the formula for the second Poisson brackets of the variables $u_{1}$. For $d \neq 1$ it has a Virasoro-type structure

$$
\left\{u_{1}(x), u_{1}(y)\right\}_{2}=\frac{1-d}{2}\left[u_{1}(x)+u_{1}(y)\right] \delta^{\prime}(x-y)+\frac{\epsilon^{2}}{2} \operatorname{tr}\left[\frac{1}{4}-\mu^{2}\right] \delta^{\prime \prime \prime}(x-y)+\mathcal{O}\left(\epsilon^{4}\right)
$$

within the $\epsilon^{2}$ approximation.

One more property is existence of a tau-function for the constructed hierarchy due to tau-symmetry (3.70). Moreover, the full hierarchy is invariant with respect to a group of symmetries generated by linear action of Virasoro operators $L_{m}, m \geq-1$, onto tau-function. 
In [22] it was formulated a problem of classification of integrable hierarchy of bihamiltonian evolutionary partial differential equations of the form

$$
\frac{\partial \mathbf{u}}{\partial t_{p}^{\alpha}}=\sum_{n \geq 0} \epsilon^{n} \mathbf{K}_{n ; \alpha, p}\left(\mathbf{u} ; \mathbf{u}_{x}, \mathbf{u}_{x x}, \ldots, \mathbf{u}^{(n+1)}\right)
$$

where $\mathbf{K}_{n ; \alpha, p}\left(\mathbf{u} ; \mathbf{u}_{x}, \mathbf{u}_{x x}, \ldots, \mathbf{u}^{(n+1)}\right)$ for any $n \geq 0$ is a polynomial in the jet variables $\mathbf{u}_{x}, \mathbf{u}_{x x}, \ldots, \mathbf{u}^{(n+1)}$ of graded degree $n+1$, i.e.,

$$
\mathbf{K}_{n ; \alpha, p}\left(\mathbf{u} ; \lambda \mathbf{u}_{x}, \lambda^{2} \mathbf{u}_{x x}, \ldots, \lambda^{n+1} \mathbf{u}^{(n+1)}\right)=\lambda^{n+1} \mathbf{K}_{n ; \alpha, p}\left(\mathbf{u} ; \mathbf{u}_{x}, \mathbf{u}_{x x}, \ldots, \mathbf{u}^{(n+1)}\right)
$$

for any $\lambda$ satisfying the tau-symmetry condition and also invariant with respect to symmetries generated by a linear action onto tau-functions of Virasoro operators of the above form. It was shown that such a hierarchy, under an additional assumption of semisimplicitly of the linear operator $\mathbf{u}_{x} \mapsto \mathbf{K}_{0 ; \alpha, p}\left(\mathbf{u} ; \mathbf{u}_{x}\right)$ for at least one pair of indices $\alpha, p$ and also a certain condition of nondegeneracy of the $\epsilon=0$ term of the first Poisson bracket, is equivalent, up to a Miura-type transformation

$$
\mathbf{u} \mapsto \mathbf{F}_{0}(\mathbf{u})+\sum_{n \geq 1} \epsilon^{n} \mathbf{F}_{n}\left(\mathbf{u} ; \mathbf{u}_{x}, \ldots, \mathbf{u}^{(n)}\right)
$$

satisfying

$$
\operatorname{det}\left(\frac{\partial F_{0}^{\alpha}(\mathbf{u})}{\partial u^{\beta}}\right) \neq 0,
$$

the graded degree of differential polynomials $\mathbf{F}_{n}\left(\mathbf{u} ; \mathbf{u}_{x}, \ldots, \mathbf{u}^{(n)}\right)$ is equal to $n$, to an integrable hierarchy of topological type constructed from a semisimple Frobenius manifold by the construction of the Theorem 3.6 (in this more general case the construction will depend on the choice of calibration, i.e., on the choice of a basis of horizontal sections of the deformed flat connection $\tilde{\nabla}$ ).

In order to complete the proposed axiomatic approach to the theory of integrable hierarchies of topological type one has to fix the problem of cancellation of denominators. Namely, like in the $\mathrm{KdV}$ case the functions $\mathcal{F}_{g}\left(\mathbf{v} ; \mathbf{v}_{x}, \mathbf{v}_{x x}, \ldots, \mathbf{v}^{(3 g-2)}\right)$ are not differential polynomials. Nevertheless the equations of the resulting integrable hierarchy of topological type proved to be polynomial in jet variables $\mathbf{v}_{x}, \mathbf{v}_{x x}$ etc. at every order of the $\epsilon$-expansion (only even powers of $\epsilon$ occur). Cancellation of denominators in the equations of the hierarchy, in their Hamiltonians and in the first Poisson bracket was proved by A.Buryak, H.Posthuma and S.Shadrin [5]. So the last problem to be fixed in order to complete the proposed axiomatic formulation of the theory of integrable hierarchies of topological type is to also prove polynomiality of the second Poisson bracket. It would also be very interesting to find a Lax representation (see (2.4) above) for the integrable hierarchies of topological type. First steps in this direction have been done in [8]. 


\section{Integrable hierarchies at the degree zero approx- imation}

In this section we consider much simpler situation of intersection theory on moduli stacks of stable maps of degree zero. In this case, under certain not very restrictive assumption about a smooth projective variety $X$ we will construct explicitly the associated integrable hierarchy involved in description of invariants of all genera. In this section we will assume that $X$ is a Fano variety with vanishing $H^{\text {odd }}(X, \mathbb{C})$ of the complex dimension

$$
d \geq 4
$$

Holomorphic maps to $X$ of degree $\beta=0$ are just maps to a point $C_{g} \rightarrow$ pt $\in X$. So, at a first glance it looks like the moduli space $\bar{M}_{g, m}(X, \beta=0)$ splits into a Cartesian product of the Deligne-Mumford moduli space $\overline{\mathcal{M}}_{g, m}$ of stable algebraic curves of genus $g$ with $m$ punctures and the variety $X$ itself. The situation becomes more delicate if we look at the virtual fundamental class of $\bar{M}_{g, m}(X, \beta=0)$.

Let us begin again with genus zero. In this case the moduli space $\bar{M}_{0, m}(X, \beta=0)$ is smooth, so, indeed,

$$
\bar{M}_{0, m}(X, \beta=0)=\overline{\mathcal{M}}_{0, m} \times X
$$

So

$$
\left\langle\tau_{p_{1}}\left(\gamma_{\alpha_{1}}\right) \ldots \tau_{p_{m}}\left(\gamma_{\alpha_{m}}\right)\right\rangle_{g=0, m, \beta=0}=\int_{\overline{\mathcal{M}}_{0, m}} \psi_{1}^{p_{1}} \ldots \psi_{m}^{p_{m}} \int_{X} \gamma_{\alpha_{1}} \ldots \gamma_{\alpha_{m}} .
$$

The Frobenius manifold $M$ in this case is trivial: the algebras on the tangent spaces to $M$ are canonically isomorphic to the cohomology ring $H^{*}(X)$. The Principal Hierarchy for such a Frobenius manifold takes the form

$$
\frac{\partial \mathbf{v}}{\partial t_{p}^{\alpha}}=\frac{\partial}{\partial x}\left(\gamma_{\alpha} \cdot \frac{\mathbf{v}^{p+1}}{(p+1) !}\right), \quad \alpha=1, \ldots, n=\operatorname{dim} H^{*}(X), \quad p \geq 0
$$

The bihamiltonian structure of this hierarchy obtained from the general prescription of the theory of Frobenius manifolds can be written in the following form

$$
\begin{aligned}
& \{\langle a, \mathbf{v}(x)\rangle,\langle b, \mathbf{v}(y)\rangle\}_{1}=\langle a, b\rangle \delta^{\prime}(x-y) \\
& \{\langle a, \mathbf{v}(x)\rangle,\langle b, \mathbf{v}(y)\rangle\}_{2}= \\
& =\left[\left\langle\left(\frac{a}{2}+\mu(a)\right) \cdot b, \mathbf{v}(x)\right\rangle+\left\langle a \cdot\left(\frac{b}{2}+\mu(b)\right), \mathbf{v}(y)\right\rangle+\left\langle a \cdot b, c_{1}(X)\right\rangle\right] \delta^{\prime}(x-y) .
\end{aligned}
$$

Here $a, b \in H^{*}(X)$ are arbitrary cohomology classes. The genus zero tau-function is given by the formula

$$
\epsilon^{2} \log \tau_{0}=\sum_{m \geq 3} \frac{1}{m(m-1)(m-2)} \sum_{p_{1}, \ldots, p_{m}} \int_{X} \frac{\mathbf{t}_{p_{1}}}{p_{1} !} \ldots \frac{\mathbf{t}_{p_{m}}}{p_{m} !}
$$


where, like above, we consider cohomology-valued time variables $\mathbf{t}_{p}=t_{p}^{\alpha} \gamma_{\alpha} \in H^{*}(X)$.

Let us now proceed to the description of higher genera.

Theorem 4.1 For $\operatorname{dim}_{\mathbb{C}} X \geq 4$ the total Gromov-Witten potential of degree zero is (log of) a tau-function of the following integrable hierarchy

$\frac{\partial \mathbf{u}}{\partial t_{p}^{\alpha}}=\frac{\partial}{\partial x}\left(\gamma_{\alpha} \cdot\left[\frac{\mathbf{u}^{p+1}}{(p+1) !}+\frac{\epsilon^{2}}{24} c_{d} \cdot\left(2 \frac{\mathbf{u}^{p-1}}{(p-1) !} \mathbf{u}_{x x}+\frac{\mathbf{u}^{p-2}}{(p-2) !} \mathbf{u}_{x}^{2}\right)-\frac{\epsilon^{2}}{24} c_{d-1} \cdot \frac{\mathbf{u}^{p-1}}{(p-1) !} \mathbf{u}_{x}^{2}\right]\right)$

where $c_{1}=c_{1}(X)$ and $c_{d-1}=c_{d-1}(X)$ are Chern classes of the tangent bundle of $X$.

The proof is based on the following

Lemma 4.2 For any smooth projective variety $X$ the genus one and degree zero GromovWitten potential is given by the following formula

$$
\mathcal{F}_{1}=\frac{1}{24}\left\langle c_{d}(X), \log \mathbf{v}_{x}\right\rangle-\frac{1}{24}\left\langle c_{d-1}(X), \mathbf{v}\right\rangle
$$

where $\mathbf{v}=\mathbf{v}(\mathbf{t})$ is the solution

$$
\mathbf{v}=\sum_{n=1}^{\infty} \frac{1}{n} \sum_{p_{1}+\cdots+p_{n}=n-1} \frac{\mathbf{t}_{p_{1}}}{p_{1} !} \ldots \frac{\mathbf{t}_{p_{n}}}{p_{n} !}
$$

to the hierarchy (4.3) (cf. formula (4.5) for the tau function of this solution).

Proof For $\beta=0$ the dimension of the "naïve" moduli space $\overline{\mathcal{M}}_{g, m} \times X$ is equal to $3 g-3+m+d$ while the dimension (3.10) of the virtual fundamental class is smaller

$$
\operatorname{dim}\left[\bar{M}_{g, m}(X, \beta=0)\right]^{\mathrm{virt}}=3 g-3+m+d-g d .
$$

The discrepancy comes from the obstruction bundle (see details in the book [42]). The following formula for the degree zero invariants of any genus is due to M.Kontsevich and Yu.I.Manin [39]

$$
\left\langle\tau_{p_{1}}\left(\gamma_{\alpha_{1}}\right) \ldots \tau_{p_{m}}\left(\gamma_{\alpha_{m}}\right)\right\rangle_{g, m, \beta=0}=\int_{\overline{\mathcal{M}}_{g, m} \times X} \psi_{1}^{p_{1}} \ldots \psi_{m}^{p_{m}} e\left(\mathcal{E}^{*} \otimes T_{X}\right) \gamma_{\alpha_{1}} \ldots \gamma_{\alpha_{m}}
$$

Here $e\left(\mathcal{E}^{*} \otimes T_{X}\right)$ is the Euler class of the obstruction bundle over $\overline{\mathcal{M}}_{g, m} \times X$. The first factor $\mathcal{E}^{*}$ is the dual to the Hodge bundle over $\overline{\mathcal{M}}_{g, m}$ whose fiber coincides with the space of holomorphic differentials on the curve $C_{g}$; the second factor is the tangent bundle of $X$. Since $d \geq 4$ the virtual dimension (4.9) is less than $m$ for $g \geq 2$. Hence 42

$$
\left\langle\tau_{p_{1}}\left(\gamma_{\alpha_{1}}\right) \ldots \tau_{p_{m}}\left(\gamma_{\alpha_{m}}\right)\right\rangle_{g, m, \beta=0}=0 \quad \text { if } \quad g \geq 2
$$


It remains to compute the Euler class of the obstruction bundle for $g=1$. An easy calculation yields

$$
e\left(\mathcal{E}^{*} \otimes T_{X}\right)=c_{d}\left(\mathcal{E}^{*} \otimes T_{X}\right)=1 \otimes c_{d}(X)-\lambda_{1} \otimes c_{d-1}(X) .
$$

Here we use the standard notation

$$
\lambda_{i}=c_{i}(\mathcal{E})
$$

for Chern classes of the Hodge bundle. Thus

$$
\begin{aligned}
& \left\langle\tau_{p_{1}}\left(\gamma_{\alpha_{1}}\right) \ldots \tau_{p_{m}}\left(\gamma_{\alpha_{m}}\right)\right\rangle_{g=1, m, \beta=0}= \\
& =\int_{\overline{\mathcal{M}}_{1, m}} \psi_{1}^{p_{1}} \ldots \psi_{m}^{p_{m}} \int_{X} c_{d}(X) \gamma_{\alpha_{1}} \ldots \gamma_{\alpha_{m}}-\int_{\overline{\mathcal{M}}_{1, m}} \lambda_{1} \psi_{1}^{p_{1}} \ldots \psi_{m}^{p_{m}} \int_{X} c_{d-1}(X) \gamma_{\alpha_{1}} \ldots \gamma_{\alpha_{m}} .
\end{aligned}
$$

Let us begin with the first term. We know (see [9]) that the generating function of the intersection numbers of the genus one $\psi$-classes is equal to

$$
\sum_{m} \frac{1}{m !} \sum_{p_{1}, \ldots, p_{m}} t_{p_{1}} \ldots t_{p_{m}} \int_{\overline{\mathcal{M}}_{1, m}} \psi_{1}^{p_{1}} \ldots \psi_{m}^{p_{m}}=\frac{1}{24} \log v_{x}(\mathbf{t})
$$

(cf. formula (2.28) above) where $v(\mathbf{t})$ is the solution (3.42), (3.43) of the dispersionles KdV hierarchy. Thus

$$
\sum_{m} \frac{1}{m !} \sum_{\left(p_{1}, \alpha_{1}\right), \ldots,\left(p_{m}, \alpha_{m}\right)} t_{p_{1}}^{\alpha_{1}} \ldots t_{p_{m}}^{\alpha_{m}} \int_{\overline{\mathcal{M}}_{1, m}} \psi_{1}^{p_{1}} \ldots \psi_{m}^{p_{m}} \int_{X} c_{d}(X) \gamma_{\alpha_{1}} \ldots \gamma_{\alpha_{m}}=\frac{1}{24}\left\langle c_{d}(X), \log \mathbf{v}_{x}(\mathbf{t})\right\rangle
$$

where

$$
\mathbf{v}(\mathbf{t})=\sum_{n=1}^{\infty} \frac{1}{n} \sum_{p_{1}+\cdots+p_{n}=n-1} \frac{\mathbf{t}_{p_{1}}}{p_{1} !} \ldots \frac{\mathbf{t}_{p_{n}}}{p_{n} !}
$$

is a particular solution to the hierarchy (4.3) constructed by a procedure similar to (3.43).

Let us now proceed to the second term in (4.13). We will use the formula

$$
\int_{\overline{\mathcal{M}}_{1, m}} \lambda_{1} \psi_{1}^{p_{1}} \ldots \psi_{m}^{p_{m}}=\left\{\begin{array}{cl}
\frac{1}{24} \frac{(m-1) !}{p_{1} ! \ldots p_{m} !}, & p_{1}+\cdots+p_{m}=m-1 \\
0, & \text { otherwise }
\end{array}\right.
$$

derived by E.Getzler and R.Pandharipande [31]. So

$$
\sum_{m} \frac{1}{m !} \sum_{\left(p_{1}, \alpha_{1}\right), \ldots,\left(p_{m}, \alpha_{m}\right)} t_{p_{1}}^{\alpha_{1}} \ldots t_{p_{m}}^{\alpha_{m}} \int_{\overline{\mathcal{M}}_{1, m}} \lambda_{1} \psi_{1}^{p_{1}} \ldots \psi_{m}^{p_{m}} \int_{X} c_{d-1}(X) \gamma_{\alpha_{1}} \ldots \gamma_{\alpha_{m}}=\frac{1}{24}\left\langle c_{d-1}(X), \mathbf{v}(\mathbf{t})\right\rangle
$$

This completes the proof of Lemma. 
Since $d \geq 4$ the higher genus terms of degree zero all vanish. That is the degree zero part of the total Gromov-Witten potential is equal to

$$
\mathcal{F}=\mathcal{F}_{0}+\epsilon^{2} \mathcal{F}_{1}
$$

According to the general scheme explained in the previous sections we have to apply the substitution

$$
v_{\alpha}=\left\langle\gamma_{\alpha}, \mathbf{v}\right\rangle \mapsto u_{\alpha}=\left\langle\gamma_{\alpha}, \mathbf{u}\right\rangle=v_{\alpha}+\epsilon^{2} \frac{\partial^{2} \mathcal{F}_{1}}{\partial x \partial t_{0}^{\alpha}}
$$

to the dispersionless hierarchy (4.3). Since

$$
\frac{\partial \mathbf{v}}{\partial t_{0}^{\alpha}}=\gamma_{\alpha} \cdot \mathbf{v}_{x}
$$

we have

$$
\frac{\partial}{\partial t_{0}^{\alpha}}\left\langle c_{d}(X), \log \mathbf{v}_{x}\right\rangle=\left\langle c_{d}(X) \cdot \gamma_{\alpha}, \frac{\mathbf{v}_{x x}}{\mathbf{v}_{x}}\right\rangle=\partial_{x}\left\langle\gamma_{\alpha}, c_{d}(X) \cdot \log \mathbf{v}_{x}\right\rangle .
$$

In a similar way

$$
\frac{\partial}{\partial t_{0}^{\alpha}}\left\langle c_{d-1}(X), \mathbf{v}\right\rangle=\partial_{x}\left\langle\gamma_{\alpha}, c_{d-1}(X) \cdot \mathbf{v}\right\rangle .
$$

So the substitution (4.20) can be written in the vector form

$$
\mathbf{v} \mapsto \mathbf{u}=\mathbf{v}+\frac{\epsilon^{2}}{24} c_{d} \cdot\left(\log \mathbf{v}_{x}\right)_{x x}-\frac{\epsilon^{2}}{24} c_{d-1} \cdot \mathbf{v}_{x x}
$$

Applying this substitution to the hierarchy (4.3) one easily arrives at the equations (4.6).

One may observe similarity of eq. (4.6) with the first two terms of expansion of equations of the $\mathrm{KdV}$ hierarchy (2.10). One major difference is that the equations of the hierarchy (4.6) truncate at the order $\epsilon^{2}$ while the $i$-th equation of the KdV hierarchy contains terms up to the order $\epsilon^{2 i}$.

It is not difficult to apply the substitution (4.21) to the bihamiltonian structure (4.4) in order to arrive at the bihamiltonian structure of the equations (4.6). The resulting bihamiltonian structure reads

$$
\begin{aligned}
& \{\langle a, \mathbf{u}(x)\rangle,\langle b, \mathbf{u}(y)\rangle\}_{1}=\langle a, b\rangle \delta^{\prime}(x-y)-\frac{\epsilon^{2}}{12}\left[\left\langle a, c_{d-1}\right\rangle\left\langle b, \gamma_{n}\right\rangle+\left\langle b, c_{d-1}\right\rangle\left\langle a, \gamma_{n}\right\rangle\right] \delta^{\prime \prime \prime}(x-y) \\
& \{\langle a, \mathbf{u}(x)\rangle,\langle b, \mathbf{u}(y)\rangle\}_{2}= \\
& =\left[\left\langle\left(\frac{a}{2}+\mu(a)\right) \cdot b, \mathbf{u}(x)\right\rangle+\left\langle a \cdot\left(\frac{b}{2}+\mu(b)\right), \mathbf{u}(y)\right\rangle+\left\langle a \cdot b, c_{1}(X)\right\rangle\right] \delta^{\prime}(x-y) \\
& -\frac{\epsilon^{2}}{12}\left[\partial_{x}\left(\left\langle a, c_{d-1}\right\rangle\left\langle b \cdot \gamma_{n}, \mathbf{u}(x)\right\rangle \delta^{\prime \prime}(x-y)\right)-\partial_{y}\left(\left\langle b, c_{d-1}\right\rangle\left\langle a \cdot \gamma_{n}, \mathbf{u}(y)\right\rangle \delta^{\prime \prime}(x-y)\right)\right] \\
& +\frac{\epsilon^{2}}{12}\left\langle a \cdot b, \frac{3-d}{2} c_{d}-c_{1} \cdot c_{d-1}\right\rangle \delta^{\prime \prime \prime}(x-y) .
\end{aligned}
$$


For $d<4$ some degree 0 intersection numbers of genus $g>1$ can be nonzero. We will consider just one very simple example of $X=\mathbf{P}^{1}$ from which the following expressions for intersection numbers of genus 2 tautological classes can be derived.

Proposition 4.3 The following formula holds true

$$
\sum_{n \geq 1} \frac{1}{n !} \sum_{k_{1}, \ldots, k_{n}} t_{k_{1}} \ldots t_{k_{n}} \int_{\overline{\mathcal{M}}_{2, n}} \psi_{1}^{k_{1}} \ldots \psi_{n}^{k_{n}} \lambda_{1}=\frac{1}{480} \frac{v_{x x x}}{v_{x}}-\frac{11}{5760} \frac{v_{x x}^{2}}{v_{x}^{2}}
$$

Here $v(\mathbf{t})$ is the solution (3.43) to the dispersionless KdV hierarchy.

Proof From the expression (3.78) for the genus $2 \mathrm{GW}$ potential for $X=\mathbf{P}^{1}$ in the degree 0 limit $q \rightarrow 0$ one obtains the expression for the generating function of the genus 2 degree 0 invariants

$$
\mathcal{F}_{2}^{0}=\frac{7}{5760} u_{x x}+\frac{11 v_{x x}^{2}}{2880 v_{x}^{2}}-\frac{v_{x x x}}{240 v_{x}} .
$$

Here $v=v(\mathbf{t}), u=u(\mathbf{t}, \mathbf{s})$ is the topological solution to the dispersionless Principal Hierarchy

$$
\begin{aligned}
& \frac{\partial v}{\partial t_{k}}=\frac{\partial}{\partial x} \frac{v^{k+1}}{(k+1) !}, \quad \frac{\partial u}{\partial t_{k}}=\frac{\partial}{\partial x}\left(\frac{v^{k}}{k !} u\right) \\
& \frac{\partial v}{\partial s_{k}}=0, \quad \frac{\partial u}{\partial s_{k}}=\frac{\partial v}{\partial t_{k}} .
\end{aligned}
$$

Like in Example 3.9 we redenote $t_{k}^{1} \rightarrow t_{k}, t_{k}^{2} \rightarrow s_{k}$. Observe that $v(\mathbf{t})$ coincides with the solution (3.43) to the dispersionless hierarchy (2.7). The function $u=u(\mathbf{t}, \mathbf{s})$ is a linear homogeneous function of $\mathbf{s}$. In our case the Euler class of the obstruction bundle equals 31

$$
e(\mathcal{E})=\lambda_{2} \otimes 1-\lambda_{1} \otimes c_{1}\left(\mathbf{P}^{1}\right)
$$

So one has the following expression for the genus 2 intersection numbers on the moduli stacks $\bar{M}_{2, n}\left(\mathbf{P}^{1}, \beta=0\right)$

$$
\begin{aligned}
& \left\langle\tau_{k_{1}}\left(\gamma_{\alpha_{1}}\right) \ldots \tau_{k_{n}}\left(\gamma_{\alpha_{n}}\right)\right\rangle_{2, n, \beta=0}= \\
& =\int_{\overline{\mathcal{M}}_{2, n}} \psi_{1}^{k_{1}} \ldots \psi_{n}^{k_{n}} \lambda_{2} \int_{\mathbf{P}^{1}} \gamma_{\alpha_{1}} \ldots \gamma_{\alpha_{n}}-\int_{\overline{\mathcal{M}}_{2, n}} \psi_{1}^{k_{1}} \ldots \psi_{n}^{k_{n}} \lambda_{1} \int_{\mathbf{P}^{1}} c_{1}\left(\mathbf{P}^{1}\right) \gamma_{\alpha_{1}} \ldots \gamma_{\alpha_{n}} .
\end{aligned}
$$

Specializing at $\gamma_{1}=\cdots=\gamma_{n}=1$ one obtains

$$
\left.\frac{\partial^{n} \mathcal{F}_{2}^{0}}{\partial t_{k_{1}} \ldots \partial t_{k_{n}}}\right|_{\mathbf{t}=\mathbf{s}=0}=\left\langle\tau_{k_{1}}(1) \ldots \tau_{k_{n}}(1)\right\rangle_{2, n, \beta=0}=-2 \int_{\overline{\mathcal{M}}_{2, n}} \psi_{1}^{k_{1}} \ldots \psi_{n}^{k_{n}} \lambda_{1} .
$$

Since the derivatives of $u$ all vanish at $\mathbf{s}=0$ the above expression together with (4.24) immediately implies (4.26). 
Remark 4.4 With the help of formula (4.24) one can easily derive also the formula of C.Faber and R.Pandharipande [27] for the following intersection numbers

$$
\int_{\overline{\mathcal{M}}_{2, n}} \psi_{1}^{k_{1}} \ldots \psi_{n}^{k_{n}} \lambda_{2}=\frac{7}{5760} \frac{(n+1) !}{k_{1} ! \ldots k_{n} !} .
$$

Note that this number equals zero unless

$$
k_{1}+\cdots+k_{n}=n+1 \text {. }
$$

To this end let us choose, say, $\gamma_{\alpha_{1}}=\omega$ and others $\gamma_{\alpha_{2}}, \ldots, \gamma_{\alpha_{n}}$ equal to 1 . The formula (4.27) together with (4.24) yield

$$
\begin{aligned}
& \int_{\overline{\mathcal{M}}_{2, n}} \psi_{1}^{k_{1}} \ldots \psi_{n}^{k_{n}} \lambda_{2}=\left.\frac{7}{5760} \partial_{x}^{2} \frac{\partial^{n} u}{\partial s_{k_{1}} \partial t_{k_{2}} \ldots \partial t_{k_{n}}}\right|_{\mathbf{t}=0}=\left.\frac{7}{5760} \partial_{x}^{2} \frac{\partial^{n} v}{\partial t_{k_{1}} \partial t_{k_{2}} \ldots \partial t_{k_{n}}}\right|_{\mathbf{t}=0}= \\
& =\frac{7}{5760}\left(\partial_{x}^{n+2} \frac{v^{k_{1}+\cdots+k_{n}+1}}{\left(k_{1}+\cdots+k_{n}+1\right) k_{1} ! \ldots k_{n} !}\right)_{\mathbf{t}=0}=\frac{7}{5760} \frac{(n+1) !}{k_{1} ! \ldots k_{n} !} .
\end{aligned}
$$

In conclusion of this section let us compare the brackets (4.22) with (3.80) considering the second Poisson bracket of the variable

$$
u_{1}=\int_{X} \mathbf{u}
$$

From (4.22) one readily obtains

$$
\left\{u_{1}(x), u_{1}(y)\right\}_{2}=\frac{1-d}{2}\left[u_{1}(x)+u_{1}(y)\right] \delta^{\prime}(x-y)+\frac{\epsilon^{2}}{12}\left[\frac{3-d}{2} n-\left\langle c_{1}, c_{d-1}\right\rangle\right] \delta^{\prime \prime \prime}(x-y) .
$$

As the expression (3.80) does not depend on $q$, one must have

$$
\frac{1}{12}\left[\frac{3-d}{2} n-\left\langle c_{1}, c_{d-1}\right\rangle\right]=\frac{1}{2} \operatorname{tr}\left[\frac{1}{4}-\mu^{2}\right] .
$$

We arrive at the following constraint on a Chern number of a variety with semisimple quantum cohomology.

Proposition 4.5 For a smooth projective variety $X$ with $H^{\text {odd }}(X)=0$ and with semisimple quantum cohomology the following equation holds true

$$
\int_{X} c_{1} \wedge c_{d-1}=\frac{3}{2} \operatorname{tr} \operatorname{deg}^{2}-\frac{\chi}{2} \operatorname{dim}(3 \operatorname{dim}+1) .
$$

Here $\chi=\chi(X)$ the Euler characteristic, $\operatorname{dim}=\operatorname{dim}_{\mathbb{C}} X$. 
Remark 4.6 After the first version of this paper was submitted to the archive the author was informed by Hsian-Hua Tseng and Burt Totaro about results of the papers [41] and [2]. In [41] it was proven that the Chern number $\int_{X} c_{1} \wedge c_{d-1}$ of any d-dimensional Kähler manifold $X$ can be expressed via its Hodge numbers. In the paper [2] stimulated by [25] it was shown that equation (4.30) for a variety $X$ with trivial odd cohomology holds true if an only if the Hodge numbers $h^{p, q}(X)$ all vanish for $p \neq q$. Vanishing of these numbers for smooth projective varieties with semisimple quantum cohomology was proven by A.Bayer and Yu.I.Manin [1]. It would be interesting to analyze the possibility of extending the construction of integrable hierarchies of topological type for smooth projective varieties with vanishing non-diagonal Hodge numbers but with non-semisimple quantum cohomology. We plan to do it in subsequent publications.

Acknowledgments. The author is grateful to G. Borot, H. Iritani, Y. Zhang for fruitful discussions and to D. Orlov for help with checking validity of the constraint (4.30) for certain Fano varieties. This work is partially supported by the European Research Council Advanced Grant FroM-PDE, by the Russian Federation Government Grant No. 2010-220-01-077 and by PRIN 2010-11 Grant "Geometric and analytic theory of Hamiltonian systems in finite and infinite dimensions" of Italian Ministry of Universities and Researches.

\section{References}

[1] A. Bayer, Yu.I. Manin, (Semi)simple exercises in quantum cohomology, In: The Fano Conference, pp. 143-173. Publisher: Univ. Torino, Turin, 2004.

[2] L. Borisov, On Betti numbers and Chern classes of varieties with trivial odd cohomology groups, arXiv:alg-geom/9703023.

[3] A. Brini, G. Carlet, P. Rossi, Integrable hierarchies and the mirror model of local $C P^{1}$, Phys. D 241 (2012) 2156-2167.

[4] A. Buryak, Dubrovin-Zhang hierarchy for the Hodge integrals, arXiv:1308.5716.

[5] A. Buryak, H. Posthuma, S.Shadrin, A polynomial bracket for the DubrovinZhang hierarchies, J. Differ. Geom. 92 (2012), no. 1, 153-185.

[6] G. Carlet, B. Dubrovin, Y. Zhang, The extended Toda hierarchy, Moscow Math. J. 4 (2004) 313-332.

[7] G. Carlet, J. van de Leur, Hirota equations for the extended bigraded Toda hierarchy and the total descendent potential of $C P^{1}$ orbifolds, J. Phys. A: Math. Theor. 46 (2013) 405205

[8] G. Carlet, J.van de Leur, H. Posthuma, S. Shadrin, Towards Lax formulation of integrable hierarchies of topological type, arXiv:1201.3928. 
[9] R. Dijkgraaf, E. Witten, Mean field theory, topological field theory, and multimatrix models, Nucl. Phys. B 342 (1990), 486-522.

[10] V. Drinfeld, V. Sokolov, Lie algebras and equations of Korteweg-de Vries type, Journal of Mathematical Sciences 30 (1985), no. 2, 1975 - 2036. Translated from Itogi Nauki i Tekhniki, Seriya Sovremennye Problemy Matematiki (Noveishie Dostizheniya) $\mathbf{2 4}$ (1984) 81-180.

[11] B. Dubrovin, Integrable systems in topological field theory, Nucl. Phys. B379 (1992) 627-689.

[12] B. Dubrovin, Geometry of 2D topological field theories, In: Integrable systems and quantum groups (Montecatini Terme, 1993), 120-348, Lecture Notes in Math., 1620, Springer, Berlin, 1996.

[13] B. Dubrovin, Flat pencils of metrics and Frobenius manifolds, In: Proceedings of 1997 Taniguchi Symposium "Integrable Systems and Algebraic Geometry", Editors M.-H. Saito, Y.Shimizu and K.Ueno, 47-72. World Scientific, 1998.

[14] B. Dubrovin, Geometry and analytic theory of Frobenius manifolds, Proceedings of ICM98, Vol. 2, 315-326.

[15] B. Dubrovin, On almost duality for Frobenius manifolds, Amer. Math. Soc. Transl. 212 (2004) 75-132.

[16] B.Dubrovin, S.-Q.Liu, Y.Zhang, On Hamiltonian perturbations of hyperbolic systems of conservation laws I: quasitriviality of bihamiltonian perturbations. Comm. Pure Appl. Math. 59 (2006) 559-615.

[17] B.Dubrovin, S.-Q.Liu, Y.Zhang, Frobenius manifolds and central invariants for the Drinfeld-Sokolov bihamiltonian structures, Adv. Math. 219 (2008) 780-837.

[18] B. Dubrovin, S.P. Novikov, The Hamiltonian formalism of one-dimensional systems of hydrodynamic type and the Bogolyubov - Whitham averaging method, Soviet Math. Dokl. 270:4 (1983), 665-669.

[19] B. Dubrovin, S.P. Novikov, Hydrodynamics of weakly deformed soliton lattices. Differential geometry and Hamiltonian theory. Uspekhi Mat. Nauk 44 (1989) 2998. English translation in Russ. Math. Surveys 44 (1989) 35-124.

[20] B. Dubrovin, Y. Zhang, Bi-Hamiltonian hierarchies in 2D TFT at one-loop approximation, Comm. Math. Phys. 198 (1998), no. 2, 311-361.

[21] B. Dubrovin, Y.Zhang, Frobenius manifolds and Virasoro constraints, Selecta Math. (N.S.) 5 (1999), 423-466.

[22] B. Dubrovin, Y.Zhang, Normal forms of integrable PDEs, Frobenius manifolds and Gromov-Witten invariants, arXiv: math/0108160 
[23] B.Dubrovin, Y.Zhang, Virasoro symmetries of the extended Toda hierarchy, Comm. Math. Phys. 250 (2004) 161-193.

[24] T. Eguchi, E. Getzler, C.-S. Xiong, Topological gravity in genus 2 with two primary fields, Adv.Theor.Math.Phys. 4 (2000) 981-998.

[25] T. Eguchi, K. Hori, C.-S. Xiong, Quantum Cohomology and Virasoro Algebra, Phys. Lett. B402 (1997) 71-8.

[26] M. Entov, L. Polterovich, Symplectic quasi-states and semi-simplicity of quantum homology, In: Toric Topology (eds. M.Harada, Y.Karshon, M.Masuda and T.Panov), 47-70, Contemporary Mathematics 460, AMS, 2008.

[27] C. Faber, R. Pandharipande, Hodge integrals and Gromov-Witten theory, Invent. math. 139 (2000), 173-199.

[28] E. Frenkel, A, Givental, T. Milanov, Soliton equations, vertex operators, and simple singularities, Funct. Anal. Other Math. 3 (2010) 47-63.

[29] H. Fan, T. Jarvis, and Y. Ruan, The Witten equation, mirror symmetry and quantum singularity theory, arXiv:0712.4025.

[30] E. Getzler, Intersection theory on $\bar{M}_{1,4}$ and elliptic Gromov-Witten invariants, J. Amer. Math. Soc. 10 (1997) 973-998.

[31] E. Getzler, R. Pandharipande, Virasoro constraints and the Chern classes of the Hodge bundle, Nuclear Physics B530 (1998) 701-714.

[32] A. B. Givental, Elliptic Gromov-Witten invariants and the generalized mirror conjecture, In: Integrable systems and algebraic geometry, Proceedings of the Taniguichi Symposium 1997, ed. M. H. Saito, Y.Shimizu and K. Ueno, World Scientific (1998), 107-155.

[33] A. Givental, Gromov-Witten invariants and quantization of quadratic Hamiltonians, Mosc. Math. J. 1 (2001) 551-568, 645.

[34] C. Hertling, Frobenius manifolds and moduli spaces for singularities, Cambridge University Press, 2002.

[35] H. Iritani, Convergence of quantum cohomology by quantum Lefschetz, J. Reine Angew. Math. 610 (2007), 29-69

[36] Y. Kawamata, Derived categories of toric varieties, Michigan Math. J. 54 (2006) 517-535.

[37] M. Kazarian, S. Lando, An algebro-geometric proof of Witten's conjecture, J. Amer. Math. Soc. 20 (2007) 1079-1089. 
[38] M. Kontsevich, Intersection theory on the moduli space of curves and the matrix Airy function, Comm. Math. Phys. 147 (1992), 1-23.

[39] M. Kontsevich, Yu.I. Manin, Relations between the correlators of the topological sigma-model coupled to gravity, Commun.Math.Phys. 196 (1998) 385-398.

[40] M. Kontsevich, Yu.I. Manin (with Appendix by R. Kaufmann), Quantum cohomology of a product, Invent. math. 124 (1996) 313-339.

[41] A.S. Libgober, J.W. Wood, Uniqueness of the complex structure on Kähler manifolds of certain homotopy type, J. Diff. Geometry 32 (1990) 139-154.

[42] Yu.I. Manin, Frobenius Manifolds, Quantum Cohomology, and Moduli Spaces, AMS Colloquium Publications, 47, Providence, Rhode Island.

[43] T. Milanov, H.-H. Tseng, The spaces of Laurent polynomials, $\mathbf{P}^{1}$-orbifolds, and integrable hierarchies, Journal für die Reine und Angewandte Mathematik 622 (2008) 189-235.

[44] M. Mirzakhani, Weil-Petersson volumes and intersection theory on the moduli space of curves, J. Amer. Math. Soc. 20 (2007) 1-23.

[45] A. Okounkov, Generating functions for intersection numbers on moduli spaces of curves, Int. Math. Res. Not. 18 (2002) 933-957.

[46] Y. Ostrover, I. Tyomkin, On the quantum homology algebra of toric Fano manifolds, Selecta Math. (N.S.) 15 (2009) 131-149.

[47] C. Teleman, The structure of 2D semi-simple field theories, Invent. Math. 188 (2012) 525-588.

[48] E. Witten, Two-dimensional gravity and intersection theory on moduli space, Surv. in Diff. Geom. 1 (1991), 243-310.

[49] E. Witten, Algebraic geometry associated with matrix models of two-dimensional gravity. In: Topological methods in modern mathematics (Stony Brook, NY, 1991), 235-269, Publish or Perish, Houston, TX (1993).

[50] Chao-Zhong Wu, Tau functions and Virasoro symmetries for Drinfeld-Sokolov hierarchies, arXiv:1203.5750. 